\title{
Enhancement of synaptic AMPA receptors depends mutually on Src and PSD-95
}

${ }^{1}$ Xiaojie Huang, ${ }^{2,3}$ Juliane M. Krüger, ${ }^{2,4}$ Anna Beroun, ${ }^{5,6}$ Weifeng Xu, ${ }^{1}$ Yan Dong, ${ }^{1,2}$ Oliver M. Schlüter

${ }^{1}$ Department of Neuroscience, University of Pittsburgh, PA, USA

${ }^{2}$ European Neuroscience Institute, Grisebachstr. 5, 37077 Göttingen, Germany

${ }^{3}$ Göttingen Graduate School for Neurosciences and Molecular Biosciences, 37077 Göttingen, Germany

${ }^{4}$ BRAINCITY, Nencki Institute of Experimental Biology, Polish Academy of Sciences, Warsaw, Poland

${ }^{5}$ Department of Neuroscience, Brown University, Providence, RI, USA

${ }^{6}$ Carney Institute for Brain Science, Brown University, Providence, RI, USA

Short title: DLG-MAGUKs regulate AMPA receptors

Corresponding Author: O. Schlüter

Corresponding Address: Department of Neuroscience, University of Pittsburgh, A210 Langley Hall, Pittsburgh, PA 15206

Email: schluter@pitt.edu

Phone: +1-412-624-1876

Number of figures: 5

Word counts: Abstract: 143

Key words: excitatory synapse; AMPA receptor; PSD-95; PSD-93; Src; DLGMAGUK; hippocampus. 


\begin{abstract}
Synaptic incorporation and removal of AMPA receptors is highly regulated to modulate the strength of synaptic transmission for long-term synaptic plasticity during brain development and associative learning. PSD-93a2 and PSD-95a, two paralogs of the DLG-MAGUK protein family of signaling scaffolds govern the synaptic incorporation and stabilization of AMPA receptors opposingly, with PSD95a promoting and PSD-93a2 inhibiting it. The associated signaling mechanisms that control the synaptic incorporation and stabilization remain elusive. Here, we used domain swapping between the antagonizing signaling scaffolds to identify the protein motifs responsible for enhancing synaptic AMPA receptors and the associated signaling protein. We narrowed down multiple motifs in the $\mathrm{N}$-terminal domain that are principally responsible for governing the enhancement by Src. Specific activation and inhibiting peptides revealed continuous activity of Src. Together, the results depict a mutual dependence of Src and PSD-95a in enhancing and maintaining synaptic AMPA receptors.
\end{abstract}




\section{Introduction}

Synaptic incorporation and removal of AMPA receptors is highly regulated to modulate the strength of synaptic transmission. This plasticity is thought to alter and shape excitatory network configurations to store information as patterns of synaptic weights and is recalled by activation of specific neuronal ensembles (Basu and Siegelbaum, 2015; Nicolelis et al., 1997). Developmental plasticity as well as Hebbian plasticity are forms of NMDA receptor-dependent long-term synaptic plasticity and are primarily expressed as changes in synaptic AMPA receptor numbers (Huganir and Nicoll, 2013; Kessels and Malinow, 2009; Malenka and Bear, 2004; Morris, 2013; Neves et al., 2008; Tsien et al., 1996; Xu et al., 2020). Despite its fundamental importance, the understanding of the signaling mechanisms that regulate AMPA receptor incorporation and removal, remain controversial and incompletely understood. While early work focused on changes at AMPA receptors themselves, later studies revealed that posttranslational modifications on the receptors appear rather permissive than instructive and triggered the concept that signaling events through signaling scaffolds in the postsynaptic density (PSD) determine the AMPA receptor content (Granger et al., 2013; Hayashi et al., 2000; Lee et al., 2003; Shi et al., 2001; Xu et al., 2008; Zamanillo et al., 1999).

The disc large (DLG)-membrane associated guanylate kinase (MAGUK) family of proteins, PSD-93/Chapsyn-110, PSD-95/synapse associated protein (SAP)90, SAP97 and SAP102, are signaling scaffolds of the PSD, which regulate AMPA receptor trafficking in the synapse during basal synaptic transmission and longterm synaptic plasticity (Béïque et al., 2006; Carlisle et al., 2008; Cuthbert et al., 
2007; Ehrlich et al., 2007; Elias et al., 2006; Liu et al., 2017; Migaud et al., 1998; Nakagawa et al., 2004; Schlüter et al., 2006; Steiner et al., 2008; Xu et al., 2008). The principal isoforms of PSD-93 and PSD-95 ( $\alpha$-isoforms) share high sequence similarity with a palmitoylated $\mathrm{N}$-terminal domain, three PDZ domains, an $\mathrm{SH} 3$ domain and a C-terminal guanylate kinase (GK) domain (Brenman et al., 1996; Cho et al., 1992; Kim et al., 1996; Krüger et al., 2013; Parker et al., 2004). In contrast, the principal isoform of SAP97 ( $\beta$-isoforms) has an N-terminal L27 domain, while SAP102 has a unique N-terminal domain with both paralogs sharing the rest of the overall domain structure (Müller et al., 1996; Müller et al., 1995; Schlüter et al., 2006). The palmitoylated a-isoforms of PSD-95 and SAP97 generally enhance synaptic incorporation of AMPA receptors (Béïque and Andrade, 2003; Elias et al., 2006; Nakagawa et al., 2004; Schlüter et al., 2006; Schnell et al., 2002b), but PSD-95a also regulates AMPA receptor removal during NMDA receptor-dependent long-term synaptic depression (Béïque and Andrade, 2003; Xu et al., 2008). Thus, the same signaling scaffold is involved in both, stabilization of synaptic AMPA receptors and their removal, indicating multiple and opposing functionalities harbored in one protein.

In the developing brain, the a-isoforms of PSD-95 and PSD-93 regulate synaptic AMPA receptor incorporation opposingly, with PSD-95a promoting and PSD-93a2 inhibiting the incorporation (Béïque et al., 2006; Carlisle et al., 2008; Favaro et al., 2018; Krüger et al., 2013). The molecular underpinnings of the opposing function are unknown and the distinct signaling mechanisms of PSD-95a to regulate synaptic AMPA receptor incorporation remain elusive. 
Members of the sarcoma (Src) family protein kinases were found to regulate longterm synaptic plasticity and the function of DLG-MAGUKs (Grant et al., 1992; Nada et al., 2003; Sato et al., 2008). Src family protein kinases reside in different activation states, dependent on ligand binding to their regulatory domains and their phosphorylation states, thus operate as graded switches (Bradshaw, 2010). Longterm synaptic potentiation (LTP) induction activates Src, which enhances NMDA receptor activities and facilitates LTP (Lu et al., 1998). PSD-95 binds to Src, but whether this binding inhibits or activates Src-mediated NMDA receptor phosphorylation is controversial (Kalia et al., 2006b; Kalia and Salter, 2003; Zhang et al., 2008; Zhao et al., 2015). Besides its effects on NMDA receptors and LTP, it remains elusive whether Src regulates synaptic AMPA receptor incorporation independent of regulating NMDA receptor function.

Here, we used domain swapping between PSD-93a2 and PSD-95a or SAP97a to identify the protein motifs responsible for enhancing synaptic AMPA receptormediated transmission. We found that multiple motifs in the $\mathrm{N}$-terminal domain of PSD-95a or SAP97 $\alpha$ are principally responsible for governing the enhancement. These motifs are associated with Src activation, leading to a mutual dependence of Src and PSD-95a in enhancing and maintaining synaptic AMPA receptors.

\section{Material and Methods}

Virus vectors and neuronal transduction: All lentiviral constructs were made from the lentiviral transfer vector FUGW (Lois et al., 2002) and its variant FHUG $+W$, which additionally contains an RNAi expression cassette driven by an H1 promoter for knocking down PSD-95 (sh95RN) or Src (shSRf) (Schlüter et al., 
2006). C-terminally eGFP-tagged PSD-93a2 and SAP97 over-expression and PSD-95-to-PSD-95a replacement constructs were described previously (Krüger et al., 2013; Schlüter et al., 2006).

Transduction of neurons in rat hippocampal slice cultures was achieved by injecting concentrated viral particles into the CA1 pyramidal cell layer on DIV 1 or DIV 2 using a Nanoject II device (Drummond Scientific) (Krüger et al., 2013).

Construction of lentiviral vectors: For the analysis of the differences in the Ntermini of PSD-93a and PSD-95a, exon 1 of PSD-93a2 was replaced by exon 1 of PSD-95 $\alpha$ in the lentiviral over-expression constructs, and vice versa, leading to the constructs PSD-93_951-9 and PSD-95_931-20, respectively. To study the differences in the N-terminus upstream of PDZ-1, the N-terminus of PSD-93a2 (amino acids aa1-104) was replaced by the N-terminus of PSD-95 $\alpha$ (aa 1-64) or $\mathrm{N}$-terminus of SAP97a (aa1-94) and vice versa, leading to the constructs PSD93_951-64 or PSD-93_971-94 and PSD-95_931-104. Exon 2 of SAP97a (aa11-65) was replaced with exon 2 of PSD-93a2 (aa22-75), and vice versa, leading to constructs SAP97_93/E222-75 and PSD-93_97/E2 $11-65$, respectively. The extended exon 3 of SAP97a (aa66-94) was replaced with that of PSD-93a2 (aa76-104), and vice versa, leading to the constructs SAP97_93/E376-104 and PSD-93_97/E366-94, respectively. To substitute F103 with Tyr in PSD-93a2, we used standard site directed mutagenesis to change the codon TTT to TAC to generate the overexpression construct PSD-93FY. In the PSD-95 wild-type replacement construct, we used site directed mutagenesis to introduce the Y63F mutation or the G59I and Y63F double mutation to generate the replacement constructs PSD-95YF and PSD-95IF, respectively. The identity of each construct was confirmed by DNA sequencing.

Hippocampal slice culture: All procedures were performed by following the procedures approved by the animal care and use committees and governmental 
agencies of the listed institutions. Organotypic hippocampal slices were prepared according to previously published procedures (Krüger et al., 2013; Schlüter et al., 2006). In short, P7-P9 Wistar rats of either sex were anesthetized with isofluorane, and the hippocampi were dissected in ice-cold sucrose cutting buffer (in mM as follows: 204 sucrose, $26 \mathrm{NaHCO}_{3}, 10$ glucose, $2.5 \mathrm{KCl}, 1 \mathrm{NaH}_{2} \mathrm{PO} 4,4 \mathrm{MgSO}_{4}, 1$ $\mathrm{CaCl}_{2}, 4$ ascorbic acid). Using a guillotine with a wire frame, 300- $\mu$ m-thick slices were cut and transferred into artificial CSF (ACSF) (containing in $\mathrm{mM}$ ) as follows: $119 \mathrm{NaCl}, 26 \mathrm{NaHCO}_{3}, 20$ glucose, $4 \mathrm{MgSO}_{4}, 2.5 \mathrm{KCl}, 1 \mathrm{NaH}_{2} \mathrm{PO}_{4}, 4 \mathrm{CaCl}_{2}$, for recovery. The slices were washed in BME (Biochrom) and plated onto MilliCell (Millipore) culture plate inserts in culture dishes containing BME-based culture medium supplemented with $20 \%$ heat-inactivated horse serum (Biochrom). The slices were incubated at $37^{\circ} \mathrm{C}$ in a $5 \% \mathrm{CO}_{2}$ environment. On DIV 1 , the slices were transferred to $34^{\circ} \mathrm{C}$, and the culture medium was refreshed.

Transduction of neurons in hippocampal slice cultures was achieved by injecting concentrated viral particles into the CA1 pyramidal cell layer on DIV 1 or DIV 2 using a Nanoject II device (Drummond Scientific).

Acute hippocampus slice preparation: 300um thick coronal acute brain slices were prepared from P18-20 mouse using a virbrating microtome (Leica VT1200S) in ice cold cutting solution containing in $\mathrm{mM}\left(119\right.$ Choline $\mathrm{Cl}, 26 \mathrm{NaHCO}_{3}, 30$ Glucose, $7 \mathrm{MgSO}_{4}, 2.5 \mathrm{KCl}, 1 \mathrm{NaH}_{2} \mathrm{PO}_{4}, 1 \mathrm{CaCl}_{2}, 1 \mathrm{Kynurenic}$ acid, $1.3 \mathrm{Na}$ ascorbate, $3 \mathrm{Na}$ pyruvate). Slices were recovered in oxygenated ACSF (119 NaCl, $26 \mathrm{NaHCO}_{3}, 20$ Glucose, $1.3 \mathrm{MgSO}_{4}, 2.5 \mathrm{KCl}, 1 \mathrm{NaH}_{2} \mathrm{PO}_{4}, 2.5 \mathrm{CaCl}_{2}$ ) at $34^{\circ} \mathrm{C}$ for 30 minutes, and then were kept at room temperature until use.

Electrophysiology: Slice culture recordings were performed 4-9 days after viral vector transduction as previously described (Krüger et al., 2013; Schlüter et al., 2006). A single slice was transferred into the recording chamber, which was constantly perfused with oxygenated ACSF (2-3 ml/min) containing in $\mathrm{mM}$ (119 
$\mathrm{NaCl}, 26 \mathrm{NaHCO}_{3}, 20$ Glucose, $\left.4 \mathrm{MgSO}_{4}, 2.5 \mathrm{KCl}, 1 \mathrm{NaH}_{2} \mathrm{PO}_{4}, 4 \mathrm{CaCl}_{2}\right) .1-5 \mu \mathrm{M}$ 2-chloroadenosine (Biolog) was added to the ACSF to reduce polysynaptic activity. $50 \mu \mathrm{M}$ picrotoxin (Ascent Scientific) was added to block inhibitory transmission and thus to isolate excitatory postsynaptic currents (EPSCs). Patch pipettes (open pipette resistance 3-6 MOhm) were filled with intracellular solution consisting of (in mM) 117.5 $\mathrm{MeSO}_{3} \mathrm{H}, 10 \mathrm{HEPES}, 17.75 \mathrm{CsCl}, 10 \mathrm{TEA}-\mathrm{Cl}, 0.25$ EGTA, 10 Glucose, $2 \mathrm{MgCl}_{2}, 4 \mathrm{Na}_{2} \mathrm{ATP}, 0.3 \mathrm{NaGTP}$ (pH 7.3, $290 \mathrm{mOsm}$ ). For pairwise recording, an infected cell and its neighboring uninfected (control) cell were simultaneously recorded using whole-cell voltage-clamp configuration. EPSCs were simultaneously evoked in paired cells by a single bipolar stimulation electrode filled with ACSF. AMPAR EPSCs were recorded at $-60 \mathrm{mV}$. For NMDAR EPSCs, EPSCs were recorded at $+40 \mathrm{mV}$, and the amplitude of NMDAR EPSCs was assessed by the amplitude of EPSCs at $60 \mathrm{msec}$ after the peak currents, a time point at which AMPAR EPSCs are mostly absent.

Acute slice recordings were performed with similar ACSF except using $1.3 \mathrm{MgSO}_{4}$ and $2.5 \mathrm{CaCl}_{2}$. Cs-gluconate based intracellular solution containing in $\mathrm{mM}$ (130 Cs gluconate, 20 HEPES, 10 EGTA, 3 TEA-Cl, 4 QX314-Cl, 4 MgATP, $0.3 \mathrm{Na}_{2} \mathrm{GTP}$ ) were used.

Signals were filtered at $4 \mathrm{kHz}$ and digitalized at $10 \mathrm{kHz}$ using an ITC-18 acquisition board (HEKA). The responses were monitored online and analyzed using customwritten acquisition and analysis software implemented in IGOR Pro (Wavemetrics). For each cell pair, a minimum of 40 sweeps was collected and averaged. The stimulation strength was adjusted such that the amplitude of AMPAR EPSCs in control cells was $\sim 100 \mathrm{pA}$. A $5 \mathrm{mV}$ hyperpolarizing step was used to monitor the input and series resistance over the recording.

The statistical analysis of electrophysiological data was done using the software Excel (Microsoft), Igor (Wavemetrics) or Prism (GraphPad). Comparison between 
pairs of control and infected cell responses were analyzed using the two-tailed paired $\mathrm{T}$ test. For none paired measures, two-tailed $\mathrm{T}$ test with unequal variance was used. Comparison between more than two constructs was performed using the ANOVA one factor test followed by Dunnett posthoc test. For Src activation peptide experiment, mixed model two-way ANOVA was used to check the difference between control and experiment group with the peptide. All data are presented as mean \pm standard error of the mean (SEM), which is shown as error bars in the graphs.

\section{Results}

\section{N-terminal domains of PSD-95 $\alpha$ and PSD-93 $\alpha 2$ transfer their differential roles in regulating AMPA receptor function}

Unlike PSD-95 $\alpha$ and SAP-97a, over-expression of the two N-terminal $\alpha$-isoforms of PSD-93 does not enhance AMPA receptor-mediated (R) EPSCs (Favaro et al., 2018; Krüger et al., 2013; Liu et al., 2014; Nakagawa et al., 2004; Schnell et al., 2002b). Conversely, gene deletion or knock-down of Dlg4 (PSD-95), but not Dlg2 (PSD-93) reduces AMPAR EPSCs (Béïque et al., 2006; Carlisle et al., 2008; Favaro et al., 2018; Krüger et al., 2013; Liu et al., 2018; Nakagawa et al., 2004). The N-terminus of PSD-95 that contained the first two PDZ domains enhances AMPAR EPSCs if over-expressed (Schnell et al., 2002b; Xu et al., 2008). To explore the structural basis for this functional difference between the $\mathrm{N}$-terminus of PSD-93a2 and PSD-95a or SAP97a, we compared the amino acid sequences and protein structure of the three DLG-MAGUKs. The first two PDZ domains are highly conserved. At the $\mathrm{N}$-terminus, the highest variability is in the exon 2 , which in Dlg4 only extends shortly over the conserved motif in $\alpha$ and $\beta$-DLG-MAGUKs that translates to KYRYQDED (Fig. 1A). Exon 2 in Dlg2 and Dlg1 (SAP97) is twice as long as in Dlg4. However, the length per se does not determine the functional 
difference, because over-expression of SAP97a enhances AMPAR function (Schlüter et al., 2006; Schnell et al., 2002b), whereas PSD-93as do not (Favaro et al., 2018; Krüger et al., 2013). However, one study implicates functional redundancy of PSD-95 and -93 in supporting synaptic AMPAR EPSCs (Elias et al., 2006).

To establish the baseline observation to compare and contrast domain swapping mutants, we over-expressed PSD-93a2 and PSD-95a, and measured AMPAR EPSCs with the dual whole cell patch-clamp procedure, simultaneously recording from a transduced CA1 pyramidal neuron and a neighboring reference neuron in rat organotypic slice cultures (Fig. 1B). This recording configuration allows the quantitative comparison of AMPAR EPSCs between the two conditions (Hayashi et al., 2000; Schlüter et al., 2006). Consistent with our previous results in mouse visual cortex and rat organotypic hippocampal slice cultures, over-expression of PSD-93a2 did not alter AMPAR EPSCs ( $n=22$; control, -83.47 \pm 8.93 pA; infected, $-88.76 \pm 10.66$ pA, p = 0.44; Fig. 1C) (Krüger et al., 2013). In contrast, overexpression of PSD-95 enhanced AMPAR EPSCs ( $n=11$; control, $-49.88 \pm 9.61$ pA; infected, -179.53 \pm 21.76 pA, p < 0.01; Fig. 1D)

As a construct of PSD-95 with the N-terminus and the first two PDZ domains enhanced AMPAR EPSCs (Schnell et al., 2002b; Xu et al., 2008), we overexpressed a counterpart construct of PSD-93 $\alpha 2$. However, the N-terminal PSD-93 construct had no such effect, indicating that the N-terminus of PSD-93 lacks motifs for enhancing AMPARs $(n=11$; control, $-60.78 \pm 4.61 p A$; infected, $-48.66 \pm 7.41$ $\mathrm{pA}, \mathrm{p}=0.14)$. To locate and characterize these critical regulatory motifs, we constructed chimeras of PSD-95a and PSD-93a2. If we swapped the N-terminal domain up to PDZ-1 of PSD-93a2 onto PSD-95, the enhancing effect of PSD-95 on AMPARs was abolished ( $n=15$; control, $-68.98 \pm 4.49$ pA; infected, $-67.70 \pm$ 7.60 pA, $p=0.88 ;$ Fig. 1E). This result echoed the result of the $\mathrm{N}$-terminal PSD-93 
construct that the AMPAR enhancing motifs in the $\mathrm{N}$-terminal domain before the first PDZ domain are absent in PSD-93 $\alpha 2$. We then did the reverse, swapping the $\mathrm{N}$-terminus of PSD-95 $\alpha$ onto PSD-93. When the full N-terminal domain (up to PDZ1) was swapped, expression of this construct increased the peak amplitude of AMPAR EPSCs ( $n=11$; control, $-40.73 \pm 4.41$ pA; infected, $-65.74 \pm 7.76 p A, p<$ 0.05; Fig. 1F). Together, these results indicated that the $\mathrm{N}$-terminal region before the first PDZ domain in PSD-93 22 does not contain the functionality of enhancing AMPAR EPSCs, whereas the N-terminal region of PSD-95 $\alpha$ does.

To further narrow down the location, we swapped the first 20 amino acids of the $\mathrm{N}$-terminus of PSD-93a2 onto PSD-95. This short sequence codes for the palmitoylation signal before the conserved KYRYQDED motif. Expression of this construct increased AMPAR EPSCs at a regulatory magnitude similar to PSD-95 $\alpha$ ( $n=10$; control, $-44.04 \pm 4.92$ pA; infected, $-96.32 \pm 19.41$ pA, $p=0.02 ;$ Fig. $1 G$ ). Conversely, swapping the very first 9 amino acids before the KYRYQDED motif from PSD-95 onto PSD-93 did not enhance AMPAR EPSCs ( $n=11$; control, $41.52 \pm 3.64$ pA; infected, $54.02 \pm 8.11$ pA, $p=0.07$; Fig. $1 \mathrm{H})$, indicating that the very $\mathrm{N}$-terminus has little contribution to the functional difference between PSD95a and PSD-93a2. Together, the above results indicated that one or more motifs between the conserved KYRYQDED and PDZ-1 were lacking in PSD-93, and these motifs were required for the enhancing effect of DLG-MAGUK $\alpha$-isoforms on AMPARs.

\section{Motifs in consecutive exons coding the $\mathrm{N}$-terminal domain contributed to the AMPAR-enhancing effect of DLG-MAGUK $\alpha$-isoforms}

Compared to PSD-95, SAP97 is more similar to PSD-93 in their N-terminal domains (Fig. 1A). Given over-expression of SAP97a upregulates AMPAR EPSCs, whereas over-expression of PSD-93a2 does not (Krüger et al., 2013; 
Schlüter et al., 2006), we reasoned that by swapping N-terminal exons between $D \lg 2$ and $D \lg 1$, we might further narrow down the critical motifs governing the $\alpha$ isoform-mediated enhancement of AMPARs. We first confirmed the enhancing effect of SAP97a (Schlüter et al., 2006). When SAP97a was over-expressed, AMPAR EPSCs were increased ( $n=23$; control, $-66.96 \pm 4.70$ pA; infected, $140.69 \pm 10.99$ pA, $p<0.01$; Fig. 2B, I). The AMPAR-enhancing effect of SAP97 $\alpha$ was transferred onto PSD-93a2 by swapping the SAP97a N-terminal domain onto PSD-93 ( $n=18$; control, $-34.99 \pm 4.07$ pA; infected, $-84.16 \pm 11.53$ pA, $p<0.01$; Fig. 2C, F), echoing the result of the PSD-95/PSD-93 N-terminal swap and further supporting the $\mathrm{N}$-terminus as the principal location of the enhancing effect.

The $\mathrm{N}$-terminal swaps so far narrowed the enhancing effect down to exons 2,3 and parts of exon 4 up to the coding region of PDZ-1. In a next step, we split the $\mathrm{N}$-terminus at the exon 2 to exon 3 boundary to either swap exon 2 or exon 3 with parts of exon 4 (referred to as extended exon 3 in the following). The PSD-93 construct with exon 2 of Dlg1 (PSD-93_97/E2 11-65) increased the peak amplitude of AMPAR EPSCs, but with a comparatively low magnitude ( $n=17$; control, -67.46 \pm 5.46 pA; infected, $-95.67 \pm 9.17$ pA, $p<0.01$; Fig. 2D). The PSD-93 construct with extended exon 3 of Dlg1 (PSD-93_97/E366-94) increased the peak amplitude of AMPAR EPSCs similar to the exon 2 swap $(n=18$; control, $-96.57 \pm 7.91 p A$; infected, $-138.24 \pm 11.95$ pA, $p<0.01$; Fig. 2E).

When comparing the magnitudes of effect of the swaps of the partial N-terminal motifs, coded by exon 2 (PSD-93_97/E211-65) or extended exon 3 (PSD93_97/E366-94) with the full N-terminal swap (PSD-93_97/N), the magnitude of enhancement of the partial swaps was smaller (PSD-93_97/N, $2.27 \pm\{+0.44$, 0.37\}; PSD-93_97/E2, $1.38 \pm\{+0.17,-0.15\} ;$ PSD-93_97/E3, $1.43 \pm\{+0.14,-0.13\}$; $F_{(2,50)}=4.29, p=0.02 ; P S D-93 \_97 / N$ vs. PSD-93_97/E2, $p=0.01 ; P S D-93 \_97 / N$ 
vs. PSD-93_97/E3, $p=0.02 ;$ Fig. 2F), indicating that both exon 2 and extended exon 3 of $D \lg 1$ contributed to the enhancement additively.

In the converse experiment, the SAP97 construct with Dlg2 exon 2 (SAP97_93/E222-75) increased the peak amplitude of AMPAR EPSCs ( $\mathrm{n}=9$; control, $-69.79 \pm 11.21 \mathrm{pA}$; infected, $-158.23 \pm 16.83 \mathrm{pA}, \mathrm{p}<0.01$; Fig. $2 \mathrm{G})$. Similarly, the SAP97 construct with Dlg2 extended exon 3 (SAP97_93/E376-104) increased the peak amplitude of AMPAR EPSCs $(n=19$; control, $-63.81 \pm 5.36$ pA; infected, $-110.28 \pm 15.29$ pA, $p<0.01$; Fig. $2 \mathrm{H}$ ). Comparison of the magnitude of effect of SAP97a with the SAP97 constructs with DIg2 exon 2 (SAP97_93/E22275) or extended exon 3 (SAP97_93/E376-104) revealed no significant difference and only swapping the D/g2 extended exon 3 exhibited a trend of smaller enhancement $\left(\mathrm{SAP} 97,2.04 \pm\{+0.20,-0.18\} ; \quad S A P 97 \_93 / E 2,2.40 \pm\{+0.67,-0.52\}\right.$ SAP97_93/E3, $1.56 \pm\{+0.26,-0.22\} ; F_{(2,48)}=1.91, p=0.16 ;$ Fig. 2I). Thus, SAP97 with either its exon 2 or extended exon 3 enhanced AMPAR EPSCs fully, while PSD-93 required both exon 2 and extended exon 3 of either PSD-95 or SAP97 for the enhancing effect (Fig. 2). We interpret this different outcome by potential additional motifs in the C-terminal domain that might cooperate with motifs in the $\mathrm{N}$-terminal domain to either enhance with the SAP97 C-terminal domain or inhibit with the PSD-93 C-terminal domain the enhancing function. Because the AMPARenhancing motif was transferred by the N-terminus, which is not part of the palmitoylation motif, the AMPAR-enhancing motif should be either one that spans over exon 2 and 3 or a complex comprising of two relatively independent parts, one in exon 2 and the other in the extended exon 3. 


\section{A single amino acid differentiated PSD-93 from PSD-95 and SAP97 in regulating AMPARs}

Our results so far narrow down that exon 2 and extended exon 3 in Dlg4 and Dlg1 code for the likely motifs that entail the enhancing effect on synaptic AMPAR function. Thus, the critical amino acid sequence or even single amino acids that mediates the AMPAR-enhancing effect should exist in exon 2 and/or extended 3 in the a-isoform of PSD-95 and SAP97, but not in PSD-93a2. To identify a motif, we searched for conserved motifs and amino acids that are shared between PSD95 $\alpha$ and SAP97 $\alpha$ and different in PSD-93a2. Exon 2 was very similar between SAP97 and PSD-93, whereas large parts were absent in PSD-95 (Fig. 1A). However, before the PDZ-1 domain in extended exon 3, there is a conserved tyrosine in PSD-95 (Y63) and SAP97 (Y93), whereas in PSD-93 this amino acid is a phenylalanine (F103). We created point mutations in PSD-93a2 and PSD-95a to test the role of this amino acid in $\alpha$-isoform-mediated enhancement of synaptic AMPA receptor function. Because these mutations may not be dominant and can be masked by multimerization with endogenous PSD-95, we used the molecular replacement strategy to overcome this potential limitation (Xu et al., 2008). We first validated this strategy. Replacing the endogenous PSD-95 by recombinant PSD$95 a$, which resulted in over-expression of functional PSD-95, increased the peak amplitude of AMPAR EPSCs $(n=26$; control, $-60.43 \pm 6.30$ pA; infected, -202.24 . $\pm 21.87 \mathrm{pA}, \mathrm{p}<0.01$; Fig. $3 \mathrm{~B}, \mathrm{E})$. These results confirmed that the molecular replacement strategy was effective as we used previously (Schlüter et al., 2006; Xu et al., 2008). Replacing endogenous PSD-95 with PSD-95a containing the Y63F mutation reduced the magnitude of PSD-95a-mediated enhancement of AMPAR EPSCs. However, the molecular replacement with the Y63F mutant still enhanced AMPAR EPSCs ( $n=21$; control, $-71.70 \pm 5.68$ pA; infected, $-147.30 \pm$ $15.53 \mathrm{pA}, \mathrm{p}<0.01$; Fig. $3 \mathrm{C}, \mathrm{E})$. In this sequence stretch of the extended exon 3 , 
another difference between PSD-95 and PSD-93 exists, isoleucine (199) in PSD93 but glycine (G59) in PSD-95. SAP97 has an alanine at this position, which is not as bulky and hydrophobic as isoleucine, and thus might be more similar to the glycine of PSD-95. We created a double mutant of PSD-95a with G59I and Y63F to test whether the isoleucine has an additional effect on the $\alpha$-isoforms. Similar to the Y63F single mutant, PSD-95 replacement with the double mutant enhanced AMPAR EPSCs ( $n=16$; control, $-80.55 \pm 7.82$ pA; infected, $-164.97 \pm 17.93$ pA, $p$ $<0.01$; Fig. 3D, E). The effect of PSD-95a WT replacement was bigger than either PSD-95 $\alpha$ with the Y63F single mutation or with the G59I Y63F double mutation, and the mutant constructs did not differ from one another (PSD-95a, $3.30 \pm\{+0.61$, $-0.51\}$; PSD-95YF, $1.97 \pm\{+0.30,-0.26\}$; PSD-95IF, $2.03 \pm\{+0.31,-0.27\} ; F_{(2,60)}=$ 3.61, $p=0.03$; PSD-95 $\alpha$ vs. PSD-95YF, $p=0.02 ;$ PSD-95 $\alpha$ vs. PSD-95IF, $p=$ 0.04; Fig. 3E). Thus, the G59 or 199 appear irrelevant for the enhancing effect. However, Y63 is critical, but other sites in the a-isoforms of DLG-MAGUKs also contribute to the enhancement of synaptic AMPAR function.

We then performed the reverse experiment. Over-expression of PSD-93a2 alone as interleaved recordings in this set of experiments had no significant effect on AMPAR EPSCs $(n=19$; control, $-81.01 \pm 6.88$ pA; infected, $-86.06 \pm 8.19 p A, p=$ 0.68; Fig. 3F). We created a mutant PSD-93a2 in which the F103 was substituted by tyrosine. Whereas over-expression of wild-type PSD-93a2 did not affect AMPAR EPSCs, over-expression of PSD-93a2 F103Y increased the peak amplitude of AMPAR EPSCs ( $n=42$; control, $-77.76 \pm 4.81$ pA; infected, $-109.58 \pm$ 6.90 pA, p < 0.01; Fig. 3G), and its effect was different from over-expression of PSD-93a2 (PSD-93a, $1.05 \pm\{+0.12,-0.11\}$; PSD-93FY, $1.42 \pm\{+0.10,-0.09\} ; p=$ 0.03; Fig. $3 \mathrm{H}$ ). These results again indicate the critical role of $Y 63$ in the $\alpha$-isoforms of DLG-MAGUKs to mediate the enhancement of synaptic AMPA receptor 
function, and again indicated that additional unidentified sequences/motifs, possibly in exon 2, are also involved in the regulation of AMPARs.

\section{SFK activating peptide required PSD-95 to enhance synaptic AMPA receptors}

The results so far demonstrated, that motifs both in exon 2 and extended exon 3 mediate the enhancement of AMPA receptors. The conserved tyrosine, which is critically involved in this enhancement, is part of a peptide sequence YEEI, when phosphorylated activates Src family kinases (Lu et al., 1998). When applied to postsynaptic neurons, its activation of Src enhances synaptic transmission (Lu et al., 1998). Furthermore, Src binds to the N-terminus of PSD-95, but not to the other DLG-MAGUKs (Kalia et al., 2006a; Kalia and Salter, 2003). Thus, we hypothesized that differential binding and/or activation of Src family kinases by the $\mathrm{N}$-terminus of the DLG-MAGUKs might cause the differences in AMPA receptor enhancement. To test this hypothesis, we used the Src family kinase activation peptide to test whether its function depends on PSD-95. Notably, this peptide activates Src in in vitro assays in absence of any other protein (Kalia et al., 2006b). Thus, the role of PSD-95 in this experiment would be to localize activated Src at the PSD. The peptide $(1 \mathrm{mM})$ was loaded into the patch pipette. 7 min after break in, AMPAR EPSCs of CA1 pyramidal neurons in acute hippocampal slices were recorded (Fig. 4 A-E). In slices from WT mice, the AMPAR EPSCs progressively increased and plateaued after 5-10 min (between group, $F_{(1,21)}=27.39, p<0.01$; group $x$ time, $F_{(10,180)}=4.17, p<0.01 ;$ Fig. 4B). In simultaneously recorded CA1 pyramidal 
neurons without peptide, the AMPAR EPSC amplitudes did not change significantly.

In neurons of PSD-95 KO mice, Src activation peptide loading did not enhance AMPAR EPSCs, the magnitude of AMPAR EPSCs was similar to the simultaneously recorded reference neuron (between group, $F_{(1,20)}=0.38, p=0.54$; group $x$ time, $\left.F_{(9,176)}=1.50, p=0.15 ; F i g .4 C\right)$, indicating that the Src family kinase enhancing effect was dependent on PSD-95. In contrast, in neurons of PSD-93 KO mice, AMPAR EPSCs were enhanced similarly by the Src family kinase activation peptide as in WT neurons (between group, $F_{(1,30)}=22.14, p<0.01$; group $x$ time, $F_{(9,240)}=2.85, p<0.01$; Fig. 4D). The most parsimonious explanation of the results is a selective scaffolding of Src by PSD-95 and not PSD-93, consistent with the selective interaction of Src with PSD-95, but not with PSD-93 (Kalia and Salter, 2003).

It was previously reported that enhancing AMPAR EPSCs is mediated by an increase in NMDA receptor function and by lowering the threshold for LTP (Lu et al., 1998). To test whether NMDA receptor activation is required for the enhancing effect of the Src family kinase activation peptide, we repeated the experiment in WT slices in the presence of the NMDA receptor antagonist D-AP5 (between group, $F_{(1,30)}=15.16, p<0.01$; group $x$ time, $F_{(9,226)}=4.28, p<0.01$; Fig. 4E). Blocking NMDA receptors did not block the effect of the activation peptide on enhancing AMPAR EPSCs, and had also no significant effect on the AMPAR EPSCs of the reference neurons. 
We conclude that the Src family kinase activation peptide enhances AMPAR EPSCs. This enhancement requires PSD-95 and is independent of NMDA receptor signaling.

\section{PSD-95 requires Src to enhance synaptic AMPA receptors}

We next tested the opposite, whether the PSD-95a-mediated enhancing effect on AMPAR EPSCs requires Src by comparing PSD-95a over-expression with or without simultaneous knock-down of Src. To generate a loss-of function of Src, we generated a lentivirus with an shRNA to knock-down Src expression that reduced Src efficiently in rat hippocampus primary cultures (Fig. 5A). We over-expressed PSD-95 $\alpha$ without shSrc in CA1 pyramidal neurons, and recorded AMPAR EPSCs in parallel from a transduced and neighboring non-transduced neuron (Fig. 5B). Over-expression of PSD-95 $\alpha$ enhanced AMPAR EPSCs ( $n=14$; control, $-63.69 \pm$ 8.80 pA; infected, $-120.32 \pm 14.97$ pA, p < 0.01; Fig. 5C, G).

Similarly, we expressed the shSrc. AMPAR EPSCs were reduced in shSrc neurons, compared to non-transduced control neurons $(n=20$; control, $-66.60 \pm$ 6.07 pA; infected, $-49.65 \pm 3.74$ pA, p < 0.01; Fig. 5D, G), indicating that basal synaptic strength requires Src. To test the interaction of Src and PSD-95, we combined the expression of shSrc and PSD-95a in a bi-cistronic lentiviral vector (Schlüter et al., 2006). AMPAR EPSCs of transduced neurons were not significantly different from those of neighboring non-transduced control neurons ( $\mathrm{n}$ = 22; control, $-74.03 \pm 9.60$ pA; infected, $-79.84 \pm 9.74$ pA, p = 0.48; Fig. 5E, G). Thus, shSrc prevented the enhancing effect of PSD-95a over-expression.

Notably, the AMPAR EPSCs with the combination of shSrc and PSD-95a overexpression were not similarly reduced below control neuron values as with shSrc alone, an effect that we have previously observed with multiple DLG-MAGUK constructs which did not enhance AMPAR EPSCS by themselves but with knock- 
down of PSD-95, rescued transmission to control neuron level (Schlüter et al., 2006; Xu et al., 2008). So far it is not clear whether it is an additive effect of shSrc reduction and PSD-95-mediated enhancement, or rather knock-down of Src prevents the further enhancement mediated by PSD-95a, thus indicating causality. To distinguish between these possibilities, we expressed PSD-93a2 together with shSrc. Indeed, despite the lack of PSD-93a2 to itself enhance AMPAR EPSCs, the combination brought synaptic transmission back to the control level $(n=11$; control, $-44.85 \pm 7.41 p A$; infected, $-45.14 \pm 6.36 p A, p=0.96$; Fig. 5F), thus behaving similarly as what we observed in previous studies, likely by an unrelated mechanism. When calculating the ratio of AMPAR EPSCs of transduced versus untransduced reference neurons, the enhancing effect of PSD-95a overexpression was significantly different from the effects of the shSrc with PSD-95a, the shSrc with PSD-93a, or the shSrc alone group, while there was no significantly different between the other groups (PSD-95, $1.94 \pm\{+0.14,-0.13\}$; shSrc, $0.77 \pm$ $\{+0.08,-0.07\} ;$ shSrc + PSD-95, $1.05 \pm\{+0.15,-0.13\}$, shSrc + PSD-93, $1.05 \pm$ $\{+0.15,-0.13\} ; F_{(3,63)}=10.33, p<0.01 ;$ PSD-95 vs. shSrc, $p<0.01 ;$ PSD-95 vs. shSrc + PSD-95, $p<0.01 ;$ shSrc + PSD-95 vs. shSrc + PSD-93, $p=1$; shSrc vs. shSrc + PSD-95, $p=0.15$; Fig. 5G). These results indicated that PSD-95a lost its enhancing effect on synaptic AMPARs when Src expression was absent, thus behaves similar to PSD-93a2. In conclusion, Src is required for both, maintaining basal synaptic transmission and for the effect of PSD-95a on enhancing AMPAR EPSCs.

\section{Continuous Src tethering to PSD-95 is required to maintain AMPAR EPSCs}

We found so far that the Src family kinase activation peptide enhanced AMPAR EPSCs already after $10 \mathrm{~min}$ (Fig. 4). We concluded that the function of PSD-95a is to tether active Src to the PSD to enhance AMPAR EPCs. Furthermore, the 
relative fast action of the peptide indicated that Src activity is under dynamic control, and might thus be continuously activated to maintain synaptic strength. To test this hypothesis, we used a dominant interfering peptide (DIP) procedure. The PSD-95 DIP (peptide sequence: DTLEAPGYELQVNGT) was previously shown to disrupt the PSD-95/Src interaction (Kalia et al., 2006b). We loaded this peptide (1 $\mathrm{mM}$ ) into the patch-pipette and recorded AMPAR EPSCs in comparison to a parallel recorded reference cells without the peptide (Fig. $5 \mathrm{H})$. We then averaged the AMPAR EPSCs after at least 20 min break in. The AMPAR EPSCs with the PSD-95 DIP were reduced compared to the reference neurons ( $n=15$; control, $85.23 \pm 9.38$ pA; DIP, $-55.89 \pm 4.54$ pA, $p<0.01$; Fig. 5I), indicating that the PSD95/Src interaction is required to maintain the strength of AMPAR EPSCs.

For control, we used the corresponding sequence of PSD-93a2 as a putative PSD93 DIP (peptide sequence: DTLDTIPYVNGT). AMPAR EPSCs of neurons loaded with the PSD-93 DIP were not significantly different compared to the simultaneously recorded reference neuron without peptide $(n=15$; control, -81.58 $\pm 9.52 \mathrm{pA}$; infected, $-81.43 \pm 11.35 \mathrm{pA}, \mathrm{p}=0.99$; Fig. 5J). Together these results indicate that the interaction of PSD-95 with Src is required to maintain the function of synaptic AMPA receptors, presumably by tethering the active Src at the PSD to maintain the tyrosine phosphorylation of substrate proteins.

\section{Discussion}

We found that enhancing AMPAR EPSCs depends mutually on PSD-95a and Src. Using domain swapping and comparative analysis of amino acids, we identified a conserved tyrosine in PSD-95 and SAP97 $\alpha$ that is a phenylalanine in PSD-93 $\alpha 2$, coded by the extended exon 3 that together with additional motifs in the $\mathrm{N}$-terminus mediated the enhancing effect (Fig. 2, 3). The tyrosine if phosphorylated is part of a known consensus Src activation peptide motif that binds to the Src SH2 domain, 
and is localized immediately downstream of a previously described Src interaction motif in PSD-95a (Fig. 1)(Kalia et al., 2006b; Lu et al., 1998). Blocking the PSD95/Src interaction with a peptide of the interaction motif during recordings reduced EPSCs, indicating that the continuous tethering of Src by PSD-95 was required to maintain synaptic strength (Fig. 5). Together these results support a model in which Src is tethered to the PSD by PSD-95a in a signaling complex, and the PSD95 Src activation motif activates Src to maintain synaptic AMPA receptor function.

\section{Differential function of DLG-MAGUK $\alpha$-isoforms}

Using N-terminal domains swapping between PSD-93a2 and PSD-95a or SAP97a, respectively, we narrowed down motifs to a single amino acid in the Nterminus that mediate part of the enhancing effect of PSD-95 $\alpha$ and SAP97 $\alpha$ on AMPAR EPSCs. The Y63 or Y93 is conserved in PSD-95a and SAP97a, respectively, while it is the F103 in PSD-93a2. The tyrosine is part of the motif YEEI which was shown, if phosphorylated in a peptide, to bind to the $\mathrm{SH} 2$ domain of Src family kinases and to activate them (Lu et al., 1998). Swapping the tyrosine and phenylalanine between PSD-95 and PSD-93 $\alpha 2$ transferred the enhancing effect with the tyrosine onto either protein (Fig. 3). The magnitude of the enhancing effect was similar to the swap of the extended exon 3 , but smaller than the full $\mathrm{N}$ terminal swap, indicating that the tyrosine is only one part of what mediates the enhancing function.

Upstream of the putative Src activation motif in PSD-95a, is a previously described motif, coded by exon 3 that also binds to the $\mathrm{SH} 2$ domain of Src, although noncanonically, as binding does not require a phospho-Tyr (Kalia et al., 2006b). A peptide of this motif decreased AMPAR EPSCs, while the corresponding peptide of PSD-93a2 did not (Fig. 5), a result that is consistent with its role as a dominant interfering peptide to prevent the PSD-95/Src interaction. However, it was 
previously shown that the PSD-95-derived peptide competes with the Src activation peptide for binding to the Src SH2 domain, thereby inhibits Src activity (Kalia et al., 2006b). But, it is not clear whether the PSD-95-derived peptide interferes with the tethering of Src to native PSD-95a by blocking the interaction domain, thus prevent binding of the activation motif in native PSD-95a to the Src $\mathrm{SH} 2$ domain, or rather the two peptides compete for Src $\mathrm{SH} 2$ binding. Whatever the scenario, our results indicate that the tyrosine of the YEEI motif enhances AMPAR EPSCs and the effect of the PSD-95 DIP supports a tethering function of the upstream motif.

Sequence comparisons of coded amino acids of exon 2 did not reveal a similar candidate as the tyrosine, coded by the extended exon 3 (Fig. 1). Thus, the function of exon 2 remains speculative. Previous studies reported that phosphorylation by the cyclin-dependent kinase 5 (cdk5) of PSD-95 reduces its interaction with Src (Zhang et al., 2008). Cdk5 is predicted to phosphorylate T19, S25, and S35 of PSD-95a (Morabito et al., 2004). However, the N-terminal cdk5 phosphorylation sites differ among PSD-95, SAP97 and PSD-93 (Fig. 1). The T19 is unique to PSD-95a, while PSD-95a S25 is conserved in SAP97a, and conversely PSD-95a S35 is conserved in PSD-93a2. Thus, there is no clear pattern of conservation between PSD-95a and SAP97a. Nevertheless, it was reported that the phosphorylation of PSD-95a T19 promotes the interaction of the $\mathrm{N}$-terminus with $\mathrm{Ca}^{2+} /$ calmodulin (Zhang et al., 2014). T19 might thus uniquely regulate PSD-95a synaptic localization by $\mathrm{Ca}^{2+} /$ calmodulin. For Src activation, the second phosphorylation site which is conserved between PSD-95a and SAP97 $\alpha$ might be critical. Future studies will need to clarify whether these sites constitute the other part for PSD-95a to enhance AMPAR EPSCs.

While the N-terminal swaps narrowed down the motifs in PSD-95a and SAP97a that mediate the enhancement of AMPAR EPSCs and let us identify the role of SrC 
in this process, a number of observations indicate that the C-terminal domain of PSD-93a2 might have an inhibitory role in AMPAR function. Either motif coded by Dlg2 exon 2 or extended exon 3 if swapped into SAP97a did not reduce the enhancing effect of mutant SAP97a (Fig. 2), indicating that the remaining Nterminal motifs in SAP97a were sufficient to enhance AMPAR EPSCs and that neither of the PSD-93 motifs contained an active inhibitory function. The sufficiency of one of the motifs for enhancement is reminiscent of PSD-95a that lacked amino acids 46 to 64 , thus most of the Src interaction and activation motif of the extended exon 3, but was still capable to enhance AMPAR EPSCs (Xu et al., 2008). Thus, if the C-terminal domain is from PSD-95a or SAP97a, one of the N-terminal halves is sufficient for enhancing AMPAR EPSCs. In contrast, if the C-terminal part is from PSD-93, N-terminal motifs coded by both Dlg1 exon 2 or extended exon 3 were required for the full enhancing effect (Fig. 2). Furthermore, if the $\mathrm{N}$-terminal domain of PSD-95a was swapped onto the C-terminal part of PSD-93, then the magnitude of enhancement was smaller as with PSD-95a over-expression (Fig. 1). We interpret this result as evidence that the C-terminal part of PSD-93 contains an active inhibitory function for synaptic AMPA receptors. Indeed, we observed that over-expression of PSD-93a2 reduced synaptic AMPA receptor function in the visual cortex but less consistently in the hippocampus (Favaro et al., 2018; Krüger et al., 2013).

\section{Role of Src in enhancing synaptic transmission}

We found that synaptic AMPAR EPSCs depend mutually on Src and PSD-95a (Fig. 4, 5). This conclusion is supported by the following results. First, the enhancing effect of the Src family kinase activation peptide was prevented in PSD$95 \mathrm{KO}$ mice, but not PSD-93 KO mice (Fig. 4). This peptide mediates the enhancing effect via Src activation, as a Src-specific inhibitory peptide blocks this 
enhancement (Lu et al., 1998). Conversely, the enhancing effect of PSD-95a overexpression was prevented by knock-down of Src (Fig. 5). Second, the Src family kinase activation peptide enhanced AMPAR EPSCs independent of NMDA receptor activation (Fig. 4), thus similar to the PSD-95a enhancing effect on AMPAR EPSCs, does not depend on NMDA receptor activation (Schlüter et al., 2006; Zhang and Lisman, 2012). Third, a peptide mimicking the PSD-95a binding site to Src reduced AMPAR EPSCs (Fig. 5). Together these results support a model in which PSD-95a tethers Src to the PSD and promotes its activation potentially via the phosphorylated YEEI Src SH2 binding motif.

A previous study reported that the enhancement of AMPAR EPSCs is mediated by Src's effect on NMDA receptors (Lu et al., 1998). The open channel blocker MK-801 blocked the enhancing effect of the phosphorylated YEEI peptide. It is not mutually exclusive that Src enhances AMPAR EPSC by facilitating NMDA receptor signaling as well as through NMDA receptor independent mechanisms. Despite that, the action of MK-801 and D-AP5 on NMDA receptors differ. It has been shown that MK-801 blocks ionotropic signaling of NMDA receptors, but not metabotropic NMDA receptor signaling, while D-AP5 blocks both (Carter and Jahr, 2016; Nabavi et al., 2013; Stein et al., 2015). Thus, Src activation for NMDA-dependent and independent enhancement of AMPA receptor function might follow different signaling pathways.

In further support of this notion, the mechanism of Src-mediated AMPA receptor enhancement appears different to the better known role of Src to regulate NMDA receptor activity during long-term synaptic potentiation (Salter and Kalia, 2004). mGluR signaling is involved in the enhancing effect of PSD-95a over-expression on AMPAR EPSCs and GluN2A/2B phosphorylation (Heidinger et al., 2002; Zhang and Lisman, 2012). However, the signaling pathways differ. mGluR1 signaling leads to Src activation and GluN2A and 2B phosphorylation, while mGluR5 
signaling was reported to be required for the enhancing function of PSD-95a on AMPAR EPSCs. G-protein-coupled receptor activation has been linked to Src activation and mGluR5 signaling to Src family kinase activation (Benquet et al., 2002; Lu et al., 1999), but whether mGluR5 signals via Src to enhance AMPAR EPSCs remains elusive.

The interpretation is further complicated by the report that PSD-95/Src interaction has a negative role on NMDA receptor phosphorylation (Kalia et al., 2006b), while another study reports that PSD-95/Src binding enhances NMDA receptor phosphorylation and synaptic expression (Zhang et al., 2008). Furthermore, contrary to this inhibitory role of PSD-95, over-expression of PSD-95a has only a minor and a rather enhancing effect on NMDAR EPSCs (Elias et al., 2006; Nakagawa et al., 2004; Schlüter et al., 2006; Schnell et al., 2002a). Thus further studies will have to identify the mechanistic differences in synaptic regulation of AMPAR and NMDAR EPSCs by Src. In conclusion, a number of signaling pathways have been identified to activate Src and enhance synaptic NMDA and AMPA receptors, whereas our results identify motifs in the N-terminus of PSD-95a to be essential for Src to enhance and maintain synaptic AMPAR function. This results support the role of PSD-95 as signaling scaffold to regulate AMPA receptor synaptic incorporation.

\section{Acknowledgement:}

We thank S. Ott-Gebauer for excellent technical assistance, the AGCT core facility for primer synthesis and DNA sequencing. This work was funded by the National Institute for Neurological Disorders and Stroke (NIH NS107604), and the Whitehall Foundation 2018-05-68 to O.M.S. The European Neuroscience Institute Göttingen 
is jointly funded by the Göttingen University Medical School and the Max Planck Society.

\section{References:}

Basu, J., and Siegelbaum, S.A. (2015). The Corticohippocampal Circuit, Synaptic Plasticity, and Memory. Cold Spring Harbor Perspectives in Biology 7.

Béïque, J.-C., and Andrade, R. (2003). PSD-95 regulates synaptic transmission and plasticity in rat cerebral cortex. Journal of physiology 546, 859-867.

Béïque, J.-C., Lin, D.-T., Kang, M.-G., Aizawa, H., Takamiya, K., and Huganir, R.L. (2006). Synapse-specific regulation of AMPA receptor function by PSD-95. Proceedings of the National Academy of Sciences of the United States of America 103, 19535-19540.

Benquet, P., Gee, C.E., and Gerber, U. (2002). Two distinct signaling pathways upregulate NMDA receptor responses via two distinct metabotropic glutamate receptor subtypes. The Journal of neuroscience 22, 9679-9686.

Bradshaw, J.M. (2010). The Src, Syk, and Tec family kinases: distinct types of molecular switches. Cellular Signalling 22, 1175-1184.

Brenman, J.E., Christopherson, K.S., Craven, S.E., McGee, A.W., and Bredt, D.S. (1996). Cloning and characterization of postsynaptic density 93, a nitric oxide synthase interacting protein. The Journal of neuroscience 16, 7407-7415.

Carlisle, H.J., Fink, A.E., Grant, S.G.N., and O'Dell, T.J. (2008). Opposing effects of PSD-93 and PSD-95 on long-term potentiation and spike timing-dependent plasticity. Journal of physiology 586, 5885-5900.

Carter, B.C., and Jahr, C.E. (2016). Postsynaptic, not presynaptic NMDA receptors are required for spike-timing-dependent LTD induction. Nature neuroscience 19, 1218-1224. 
Cho, K.O., Hunt, C.A., and Kennedy, M.B. (1992). The rat brain postsynaptic density fraction contains a homolog of the Drosophila discs-large tumor suppressor protein. Neuron 9, 929-942.

Cuthbert, P.C., Stanford, L.E., Coba, M.P., Ainge, J.A., Fink, A.E., Opazo, P., Delgado, J.Y., Komiyama, N.H., O'Dell, T.J., and Grant, S.G.N. (2007). Synapseassociated protein 102/dlgh3 couples the NMDA receptor to specific plasticity pathways and learning strategies. The Journal of neuroscience 27, 2673-2682.

Ehrlich, I., Klein, M.E., Rumpel, S., and Malinow, R. (2007). PSD-95 is required for activity-driven synapse stabilization. Proceedings of the National Academy of Sciences of the United States of America 104, 4176-4181.

Elias, G.M., Funke, L., Stein, V., Grant, S.G.N., Bredt, D.S., and Nicoll, R.A. (2006). Synapse-specific and developmentally regulated targeting of AMPA receptors by a family of MAGUK scaffolding proteins. Neuron 52, 307-320.

Favaro, P.D., Huang, X., Hosang, L., Stodieck, S., Cui, L., Liu, Y.-z., Engelhardt, K.-A., Schmitz, F., Dong, Y., Löwel, S., et al. (2018). An opposing function of paralogs in balancing developmental synapse maturation. PLoS Biology 16, e2006838.

Granger, A.J., Shi, Y., Lu, W., Cerpas, M., and Nicoll, R.A. (2013). LTP requires a reserve pool of glutamate receptors independent of subunit type. Nature 493, 495500 .

Grant, S.G., O'Dell, T.J., Karl, K.A., Stein, P.L., Soriano, P., and Kandel, E.R. (1992). Impaired long-term potentiation, spatial learning, and hippocampal development in fyn mutant mice. Science 258, 1903-1910.

Hayashi, Y., Shi, S.H., Esteban, J.A., Piccini, A., Poncer, J.C., and Malinow, R. (2000). Driving AMPA receptors into synapses by LTP and CaMKII: requirement for GluR1 and PDZ domain interaction. Science 287, 2262-2267.

Heidinger, V., Manzerra, P., Wang, X.Q., Strasser, U., Yu, S.-P., Choi, D.W., and Behrens, M.M. (2002). Metabotropic glutamate receptor 1-induced upregulation of 
NMDA receptor current: mediation through the Pyk2/Src-family kinase pathway in cortical neurons. The Journal of neuroscience 22, 5452-5461.

Huganir, Richard L., and Nicoll, Roger A. (2013). AMPARs and Synaptic Plasticity: The Last 25 Years. Neuron 80, 704-717.

Kalia, L.V., Pitcher, G.M., Pelkey, K.A., and Salter, M.W. (2006a). PSD-95 is a negative regulator of the tyrosine kinase Src in the NMDA receptor complex. EMBO J 25, 4971-4982.

Kalia, L.V., Pitcher, G.M., Pelkey, K.A., and Salter, M.W. (2006b). PSD-95 is a negative regulator of the tyrosine kinase Src in the NMDA receptor complex. The EMBO journal 25, 4971-4982.

Kalia, L.V., and Salter, M.W. (2003). Interactions between Src family protein tyrosine kinases and PSD-95. Neuropharmacology 45, 720-728.

Kessels, H.W., and Malinow, R. (2009). Synaptic AMPA receptor plasticity and behavior. Neuron 61, 340-350.

Kim, E., Cho, K.O., Rothschild, A., and Sheng, M. (1996). Heteromultimerization and NMDA receptor-clustering activity of Chapsyn-110, a member of the PSD-95 family of proteins. Neuron 17, 103-113.

Krüger, J.M., Favaro, P.D., Liu, M., Kitlinska, A., Huang, X., Raabe, M., Akad, D.S., Liu, Y., Urlaub, H., Dong, Y., et al. (2013). Differential Roles of Postsynaptic Density-93 Isoforms in Regulating Synaptic Transmission. The Journal of neuroscience 33, 15504-15517.

Lee, H.-K., Takamiya, K., Han, J.-S., Man, H., Kim, C.-H., Rumbaugh, G., Yu, S., Ding, L., He, C., Petralia, R.S., et al. (2003). Phosphorylation of the AMPA receptor GluR1 subunit is required for synaptic plasticity and retention of spatial memory. Cell 112, 631-643.

Liu, M., Lewis, L.D., Shi, R., Brown, E.N., and Xu, W. (2014). Differential requirement for NMDAR activity in SAP97 $\beta$-mediated regulation of the number and strength of glutamatergic AMPAR-containing synapses. Journal of neurophysiology $111,648-658$. 
Liu, M., Shi, R., Hwang, H., Han, K.S., Wong, M.H., Ren, X., Lewis, L.D., Brown, E.N., and Xu, W. (2018). SAP102 regulates synaptic AMPAR function through a CNIH-2-dependent mechanism. Journal of neurophysiology 120, 1578-1586.

Liu, Y., Cui, L., Schwarz, M.K., Dong, Y., and Schlüter, O.M. (2017). Adrenergic Gate Release for Spike Timing-Dependent Synaptic Potentiation. Neuron 93, 394408.

Lois, C., Hong, E.J., Pease, S., Brown, E.J., and Baltimore, D. (2002). Germline transmission and tissue-specific expression of transgenes delivered by lentiviral vectors. Science 295, 868-872.

Lu, W.Y., Xiong, Z.G., Lei, S., Orser, B.A., Dudek, E., Browning, M.D., and MacDonald, J.F. (1999). G-protein-coupled receptors act via protein kinase C and Src to regulate NMDA receptors. Nature neuroscience 2, 331-338.

Lu, Y.M., Roder, J.C., Davidow, J., and Salter, M.W. (1998). Src activation in the induction of long-term potentiation in CA1 hippocampal neurons. Science 279, 1363-1367.

Malenka, R.C., and Bear, M.F. (2004). LTP and LTD: an embarrassment of riches. Neuron 44, 5-21.

Migaud, M., Charlesworth, P., Dempster, M., Webster, L.C., Watabe, A.M., Makhinson, M., He, Y., Ramsay, M.F., Morris, R.G.M., Morrison, J.H., et al. (1998). Enhanced long-term potentiation and impaired learning in mice with mutant postsynaptic density-95 protein. Nature 396, 433-439.

Morabito, M.A., Sheng, M., and Tsai, L.-H. (2004). Cyclin-dependent kinase 5 phosphorylates the $\mathrm{N}$-terminal domain of the postsynaptic density protein PSD-95 in neurons. The Journal of neuroscience $24,865-876$.

Morris, R.G.M. (2013). NMDA receptors and memory encoding. Neuropharmacology 74, 32-40.

Müller, B.M., Kistner, U., Kindler, S., Chung, W.J., Kuhlendahl, S., Fenster, S.D., Lau, L.F., Veh, R.W., Huganir, R.L., Gundelfinger, E.D., et al. (1996). SAP102, a 
novel postsynaptic protein that interacts with NMDA receptor complexes in vivo. Neuron 17, 255-265.

Müller, B.M., Kistner, U., Veh, R.W., Cases-Langhoff, C., Becker, B., Gundelfinger, E.D., and Garner, C.C. (1995). Molecular characterization and spatial distribution of SAP97, a novel presynaptic protein homologous to SAP90 and the Drosophila discs-large tumor suppressor protein. The Journal of neuroscience 15, 2354-2366. Nabavi, S., Kessels, H.W., Alfonso, S., Aow, J., Fox, R., and Malinow, R. (2013). Metabotropic NMDA receptor function is required for NMDA receptor-dependent long-term depression. Proceedings of the National Academy of Sciences of the United States of America 110, 4027-4032.

Nada, S., Shima, T., Yanai, H., Husi, H., Grant, S.G.N., Okada, M., and Akiyama, T. (2003). Identification of PSD-93 as a substrate for the Src family tyrosine kinase Fyn. The Journal of biological chemistry 278, 47610-47621.

Nakagawa, T., Futai, K., Lashuel, H.A., Lo, I., Okamoto, K.-I., Walz, T., Hayashi, Y., and Sheng, M. (2004). Quaternary structure, protein dynamics, and synaptic function of SAP97 controlled by L27 domain interactions. Neuron 44, 453-467.

Neves, G., Cooke, S.F., and Bliss, T.V.P. (2008). Synaptic plasticity, memory and the hippocampus: a neural network approach to causality. Nature reviews Neuroscience 9, 65-75.

Nicolelis, M.A., Fanselow, E.E., and Ghazanfar, A.A. (1997). Hebb's dream: the resurgence of cell assemblies. Neuron 19, 219-221.

Parker, M.J., Zhao, S., Bredt, D.S., Sanes, J.R., and Feng, G. (2004). PSD93 regulates synaptic stability at neuronal cholinergic synapses. The Journal of neuroscience $24,378-388$.

Salter, M.W., and Kalia, L.V. (2004). Src kinases: a hub for NMDA receptor regulation. Nat Rev Neurosci 5, 317-328.

Sato, Y., Tao, Y.-X., Su, Q., and Johns, R.A. (2008). Post-synaptic density-93 mediates tyrosine-phosphorylation of the N-methyl-d-aspartate receptors. Neuroscience $153,700-708$. 
Schlüter, O.M., Xu, W., and Malenka, R.C. (2006). Alternative N-terminal domains of PSD-95 and SAP97 govern activity-dependent regulation of synaptic AMPA receptor function. Neuron 51, 99-111.

Schnell, E., Sizemore, M., Karimzadegan, S., Chen, L., Bredt, D.S., and Nicoll, R.A. (2002a). Direct interactions between PSD-95 and stargazin control synaptic AMPA receptor number. PNAS 99, 13902-13907.

Schnell, E., Sizemore, M., Karimzadegan, S., Chen, L., Bredt, D.S., and Nicoll, R.A. (2002b). Direct interactions between PSD-95 and stargazin control synaptic AMPA receptor number. Proceedings of the National Academy of Sciences of the United States of America 99, 13902-13907.

Shi, S., Hayashi, Y., Esteban, J.A., and Malinow, R. (2001). Subunit-specific rules governing AMPA receptor trafficking to synapses in hippocampal pyramidal neurons. Cell 105, 331-343.

Stein, I.S., Gray, J.A., and Zito, K. (2015). Non-lonotropic NMDA Receptor Signaling Drives Activity-Induced Dendritic Spine Shrinkage. The Journal of neuroscience $35,12303-12308$.

Steiner, P., Higley, M.J., Xu, W., Czervionke, B.L., Malenka, R.C., and Sabatini, B.L. (2008). Destabilization of the postsynaptic density by PSD-95 serine 73 phosphorylation inhibits spine growth and synaptic plasticity. Neuron 60, 788-802.

Tsien, J.Z., Huerta, P.T., and Tonegawa, S. (1996). The essential role of hippocampal CA1 NMDA receptor-dependent synaptic plasticity in spatial memory. Cell 87, 1327-1338.

Xu, W., Löwel, S., and Schlüter, O.M. (2020). Silent Synapse-Based Mechanisms of Critical Period Plasticity. Frontiers in cellular neuroscience 14, 213.

Xu, W., Schlüter, O.M., Steiner, P., Czervionke, B.L., Sabatini, B.L., and Malenka, R.C. (2008). Molecular Dissociation of the Role of PSD-95 in Regulating Synaptic Strength and LTD. Neuron 57, 248-262.

Zamanillo, D., Sprengel, R., Hvalby, O., Jensen, V., Burnashev, N., Rozov, A., Kaiser, K.M., Köster, H.J., Borchardt, T., Worley, P., et al. (1999). Importance of 
AMPA receptors for hippocampal synaptic plasticity but not for spatial learning. Science 284, 1805-1811.

Zhang, P., and Lisman, J.E. (2012). Activity-dependent regulation of synaptic strength by PSD-95 in CA1 neurons. Journal of neurophysiology 107, 1058-1066.

Zhang, S., Edelmann, L., Liu, J., Crandall, J.E., and Morabito, M.A. (2008). Cdk5 regulates the phosphorylation of tyrosine 1472 NR2B and the surface expression of NMDA receptors. The Journal of neuroscience $28,415-424$.

Zhang, Y., Matt, L., Patriarchi, T., Malik, Z.A., Chowdhury, D., Park, D.K., Renieri, A., Ames, J.B., and Hell, J.W. (2014). Capping of the N-terminus of PSD-95 by calmodulin triggers its postsynaptic release. The EMBO journal 33, 1341-1353.

Zhao, C., Du, C.-P., Peng, Y., Xu, Z., Sun, C.-C., Liu, Y., and Hou, X.-Y. (2015). The upregulation of NR2A-containing N-methyl-D-aspartate receptor function by tyrosine phosphorylation of postsynaptic density 95 via facilitating Src/proline-rich tyrosine kinase 2 activation. Molecular Neurobiology 51, 500-511. 


\section{Figure Legends:}

Figure 1: N-terminal domains of PSD-93a2 and PSD-95a mediate specific function on AMPAR EPSCs. (A) Alignment of N-terminal amino acid sequence of PSD-93a2, PSD-95a, and SAP97 . Exon borders are indicated, and a conserved $Y$ in PSD-95a and SAP97a, while a F in PSD-93a2 highlighted in red. Domain structure of the DLG-MAGUKs is depicted on top. (B) Schematic drawing of the recording configuration of organotypic slices from rat hippocampus. (C-H) Amplitude of AMPAR EPSCs of CA1 pyramidal neurons expressing eGFP-tagged (inf) (C) PSD-93a2, (D) PSD-95a, (E) PSD-95 with PSD-93a2 N-terminal domain, (F) PSD-93 with PSD-95a N-terminal domain (G) PSD-95 with PSD-93a2 palmitoylation motif, and (H) PSD-93 with PSD-95a palmitoylation motif as lentiviral constructs are plotted against those of simultaneously recorded uninfected neighboring control neurons (ctrl). In this and all subsequent panels: gray symbols linked by a line represent single pairs of recordings; red symbols depict mean \pm SEM, p values calculated by paired t-test. Insets in each panel depict sample traces of control (black) and transduced neurons (green), Scale bar: $25 \mathrm{pA}, 20 \mathrm{~ms}$.$) .$

Figure 2: Multiple motifs in N-terminal domain contribute to the a-type enhancement. (A) Schematic drawing of the recording configuration of organotypic slices from rat hippocampus. (B-E,G,H) Amplitude of AMPAR EPSCs of CA1 pyramidal neurons expressing eGFP-tagged (B) SAP97a, (C) PSD-93 with SAP97a N-terminal domain (D) PSD-93 with SAP97a exon 2, (E) PSD-93 with SAP97a exon 3. (G) SAP97 with PSD-93a2 exon 2, and (H) SAP97 with PSD$93 \alpha 2$ exon 3 , as lentiviral constructs are plotted against those of simultaneously recorded uninfected neighboring neurons in hippocampal slice cultures. $(F, I)$ Summary graph of the comparison between indicated experimental groups of 
AMPAR EPSC amplitude ratios between transduced (inf) and control neuron (uninf). ANOVA test: * $p<0.05$.

Figure 3: A single amino acid substitution of phenylalanine to tyrosine contributes to the a-type enhancement. (A) Schematic drawing of the recording configuration of organotypic slices from rat hippocampus. (B-D, F, G) Amplitude of AMPAR EPSCs of CA1 pyramidal neurons expressing eGFP-tagged (B) PSD-95a wildtype replacement, (C) PSD-95 Y63F replacement, (D) PSD-95 G59I Y63F replacement, (F) PSD-93a2 over-expression, and (G) PSD-93 F103Y overexpression as lentiviral constructs are plotted against those of simultaneously recorded uninfected neighboring neurons (ctrl). (E, H) Summary graph of the comparison between indicated experimental groups of AMPAR EPSC amplitude ratios between transduced (inf) and control neuron (uninf). ANOVA or T-test: * $p<$ 0.05 .

Figure 4: Enhancing function of Src on AMPAR EPSCs requires PSD-95. (A) Schematic drawing of the recording configuration in mouse hippocampal acute slices with control CA1 pyramidal neuron (black) and neighboring CA1 pyramidal neuron with Src activation peptide in intracellular solution (red). (B-E) Normalized AMPAR EPSC in control (black) neuron (B) WT, (C) PSD-95 KO, (D) PSD-93 KO and $(E)$ with $50 \mu \mathrm{M}$ APV in recording ACSF, and the corresponding neuron with SFK activation peptide (red). Inset shows the sample trace of control neuron EPSC at 7.5 minute (grey) and 30 minute (black), with Src activated neuron EPSC at 7.5 minute (pink) and 30 minute (red). mixed model two-way ANOVA: ** $p<0.01$ 
Figure 5: Loss-of Src or peptide disrupting PSD-95/Src interaction inhibit AMPAR EPSCs. (A) Western blot of cell lysates of rat primary hippocampal neuron culture with knockdown of Src versus GFP control neurons. (B) Schematic drawing of the recording configuration of organotypic slices from rat hippocampus. (C-F) Amplitude of AMPAR EPSCs of CA1 pyramidal neurons expressing eGFP-tagged (C) PSD-95a, (D) shRNA against Src, (E) shSrc with PSD-95a over-expression, and (F) shSrc with PSD-93a2 over-expression as lentiviral constructs are plotted against those of simultaneously recorded uninfected neighboring CA1 pyramidal neurons. (G) Summary graph of the comparison between indicated experimental groups of AMPAR EPSC amplitude ratios between transduced (inf) and control neuron (uninf). ANOVA test: * $p<$ 0.05. (H) Schematic drawing of the recording configuration in acute slices of mouse hippocampus with control CA1 neuron (black) and neuron with peptide in the intracellular solution (red). (I-J) Amplitude of AMPAR EPSCs of CA1 pyramidal neurons with $(\mathrm{I})$ dominant interfering peptide of PSD-95 and (J) corresponding PSD-93 peptide in the intracellular solution are plotted against those of simultaneously recorded neighboring control CA1 pyramidal neurons. Insets show sample trace of control neuron (black) and neuron with peptide (red). 
bioRxiv preprint doi: httpos://doi.org/10.1101/2020.10.02.323568; this versīion posted Qctober 2, 2020. The copyright holder for this preprint (which was not certified bypeer review) is the author/funder. All rights reservēä.-No reuse allowed without permission.

B

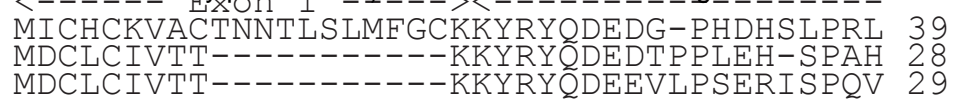

---- ExOn $2--------------------><---$

$\begin{array}{ll}\text { THEVRGPELVHVSEKNLSQIENVHGYVLQSHISPLKASPA } & 79 \\ \text { LPNO-- } & 36\end{array}$ PNEVLGPELVHVSEKSLSEIENVHGFVSHSHISPIKANPP 69

- ExOn $3----><---$ ExOn 4 - I-START PDZ1 PIIVNTDTLDTIPY---VNGTEIEYEFEEITL

PVLVNTDSLETPTY---VNGTDADYEYEEITL

C

PSD-93a2

D

PSD-95a
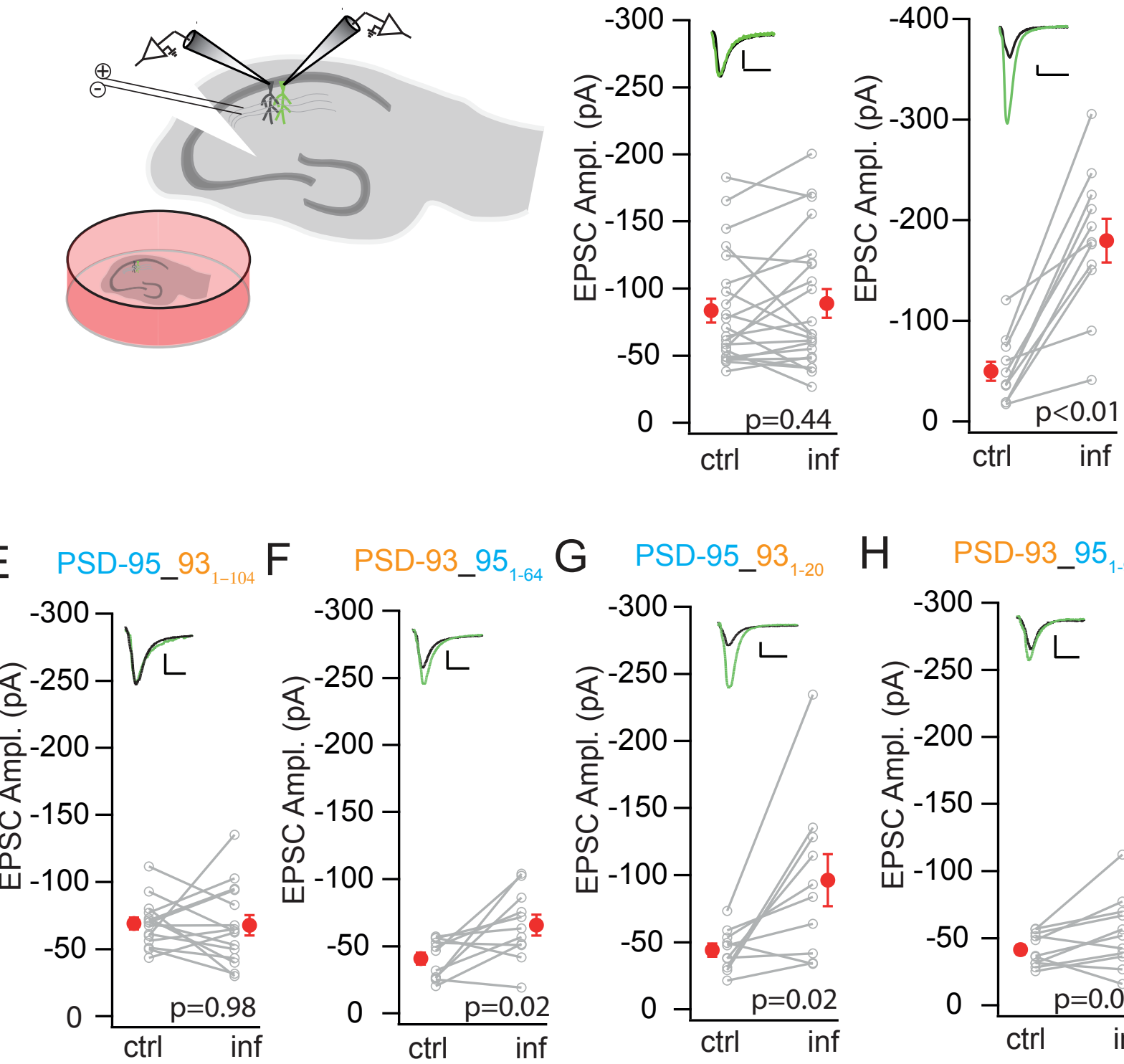

G PSD-95_93

H PSD-93_95
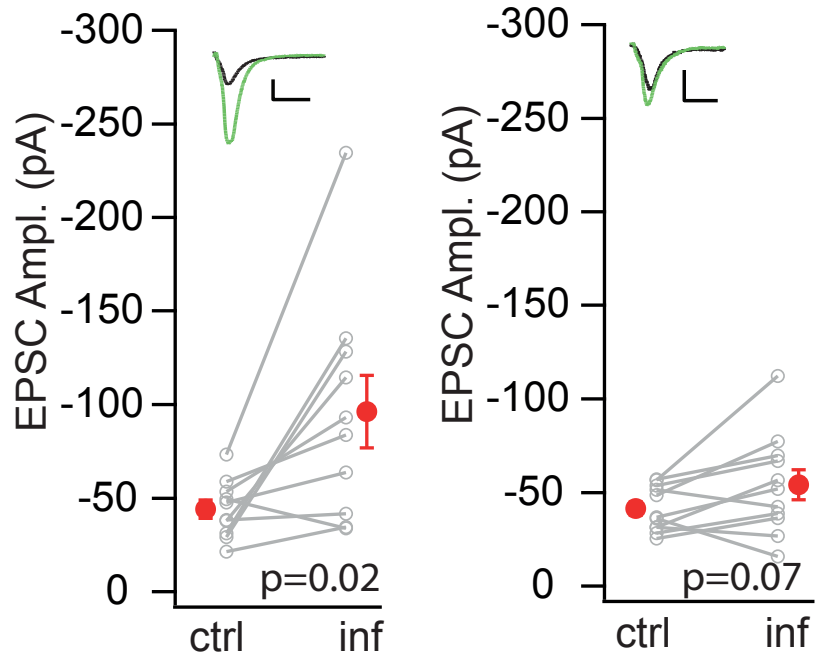

Fig. 1 
bioRxiv preprint doi: https://doi.org/10.1101/2020.10.02.323568; this version posted October 2, 2020. The copyright holder for this preprint (which was not certified by peer review) is the author/funder. All rights reserved. No reuse allowed without permission.

A
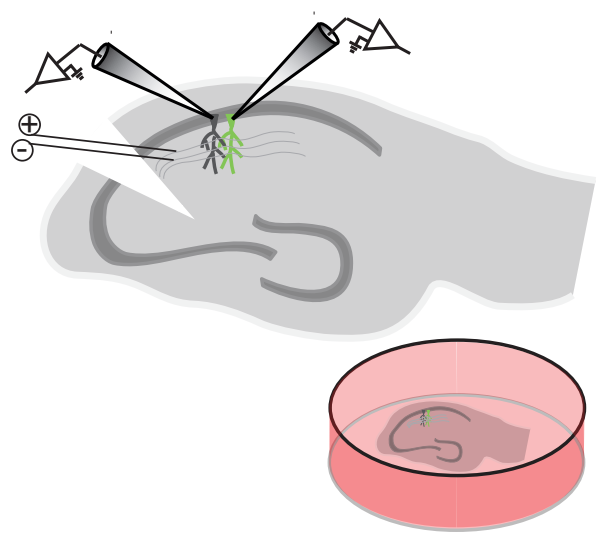

B SAP97a

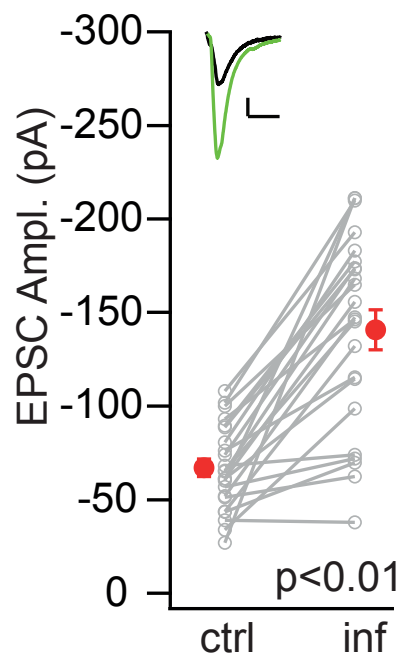

C PSD-93_97 $\mathrm{N}_{1-94}$

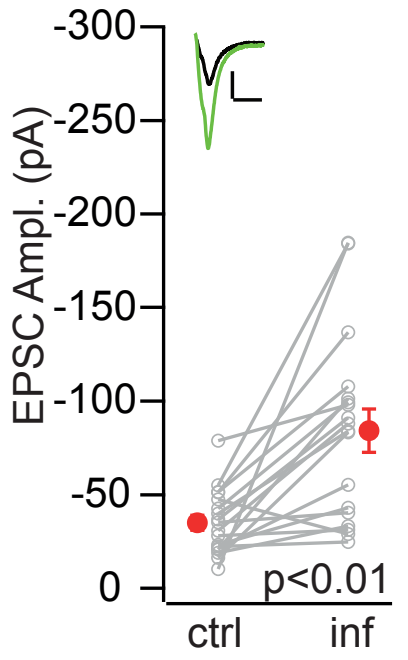

D PSD-93_97/E2 ${ }_{11-65} E$ PSD-93_97/E3 ${ }_{66-94} F$
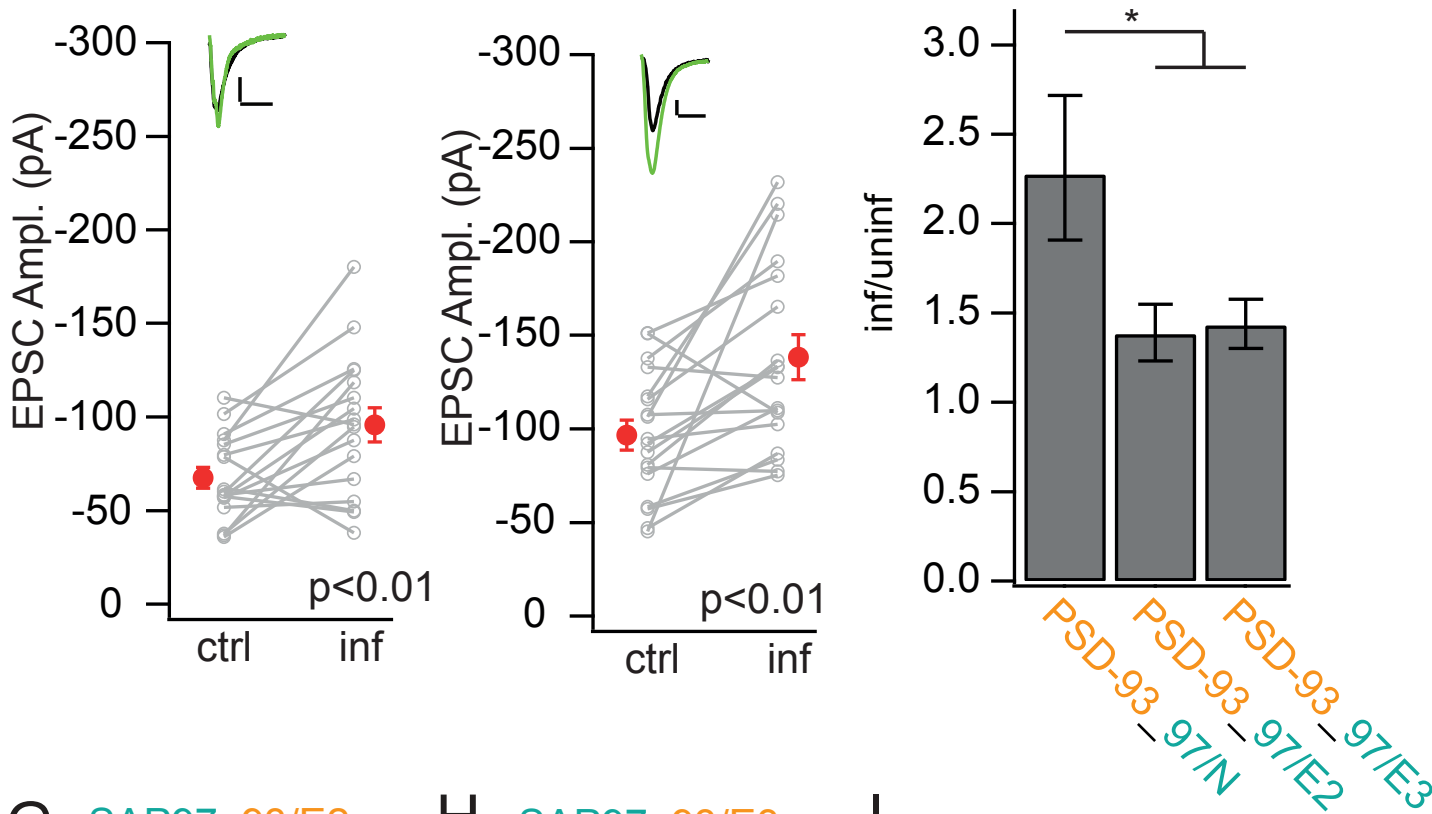

G SAP97_93/E2 $22-75$ H SAP97_93/E3 ${ }_{76-104}$ |
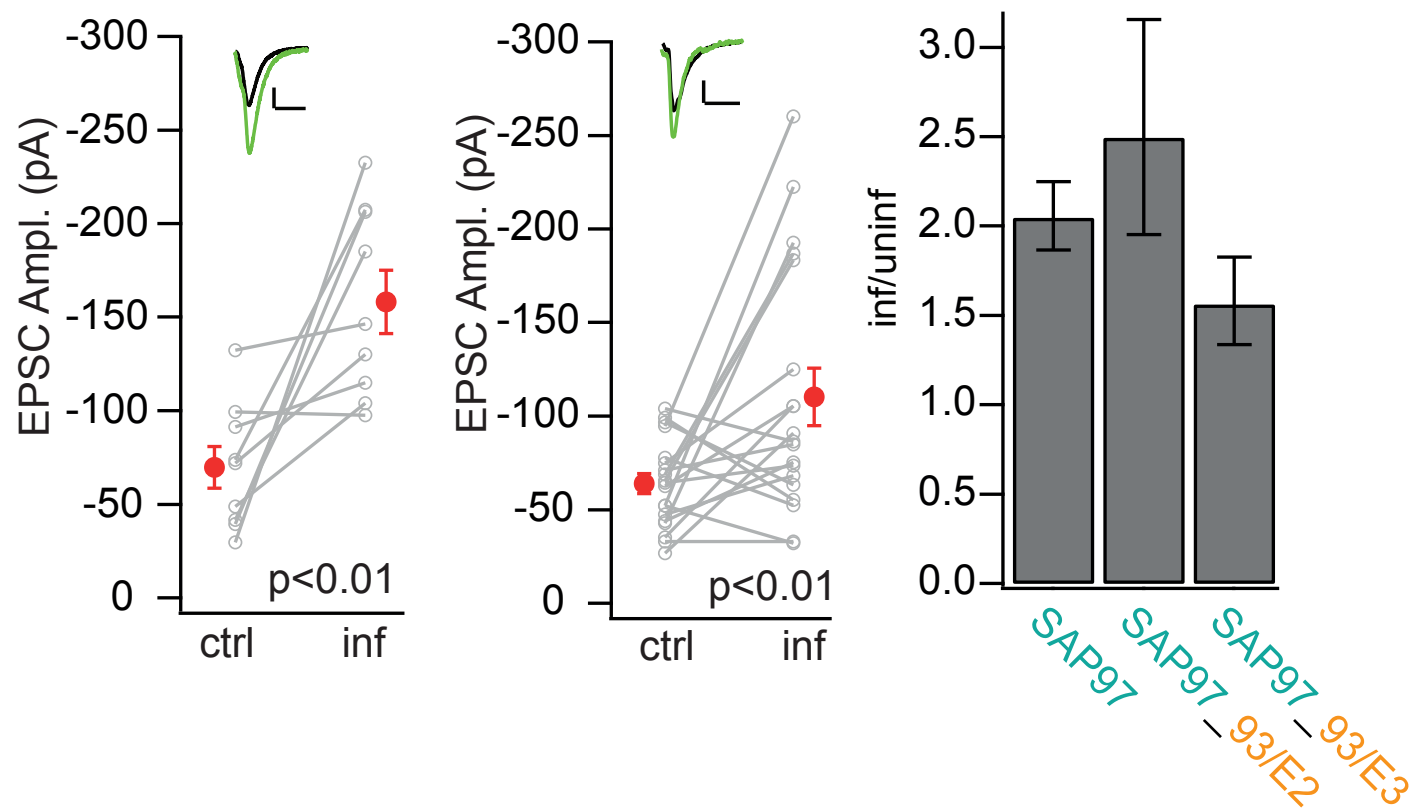

Fig. 2 

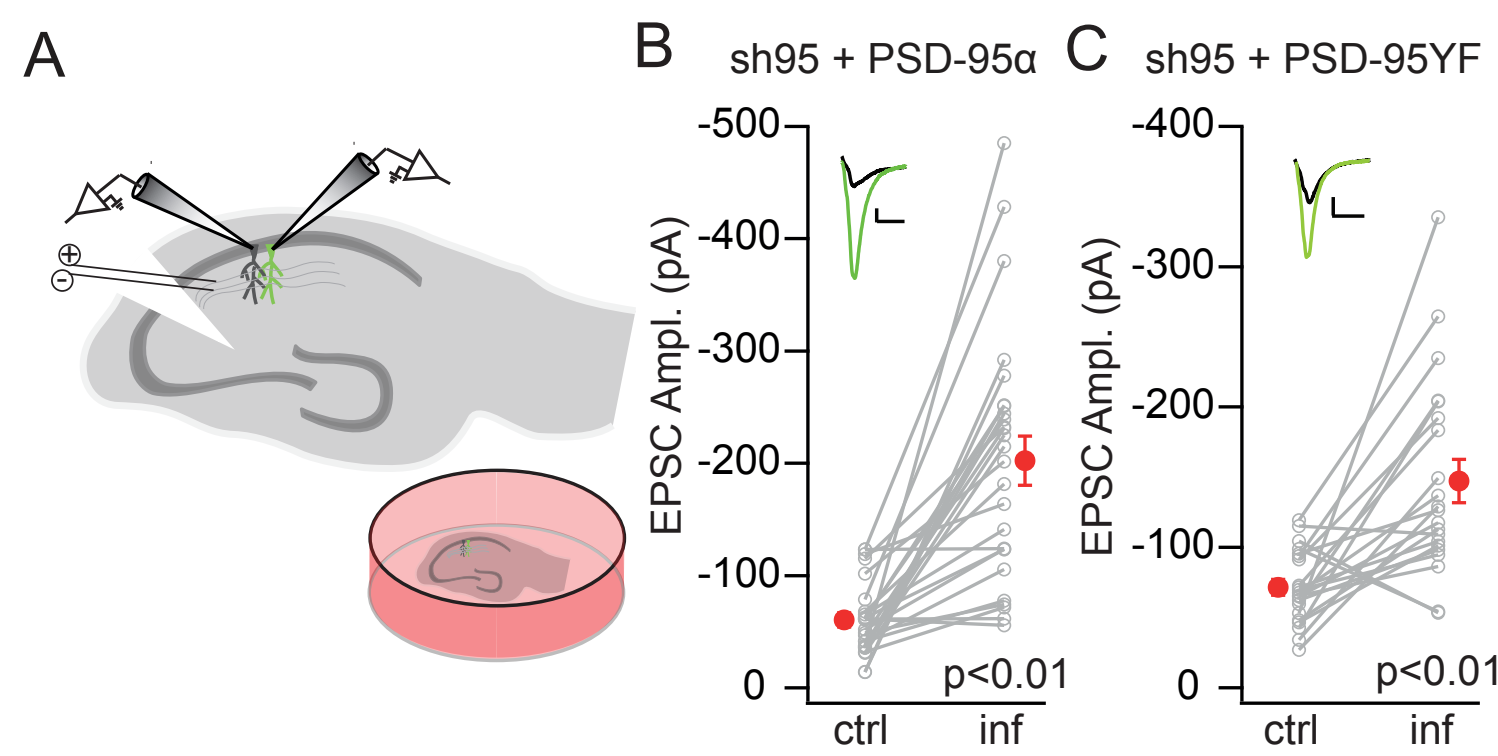

D sh95 + PSD-95IF

$\mathrm{E}$

F PSD-93a2 G

$\mathrm{G} \quad$ PSD-93FY $\mathrm{H}$
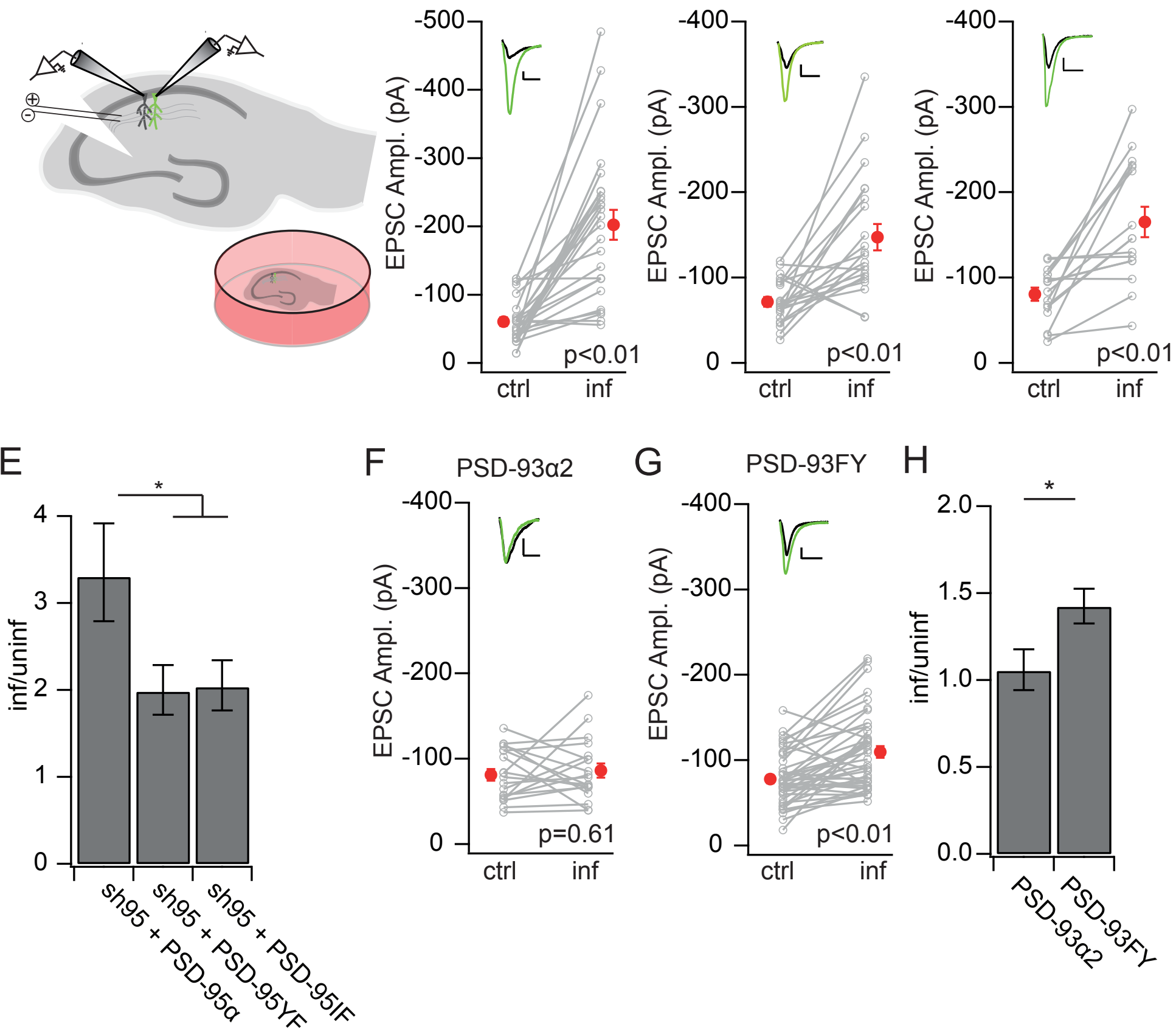

Fig. 3 
A

bioRxiv prepkint doi: http///doi.org/10.1101/2020.10.02.323568; this version posted October 2, 2020. The copyright holder for this preprint Sunkich was nd(certified by peer review) is the author/funder. All rights reserved. No reuse allowed without permission.

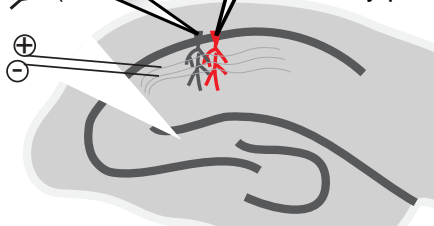

B

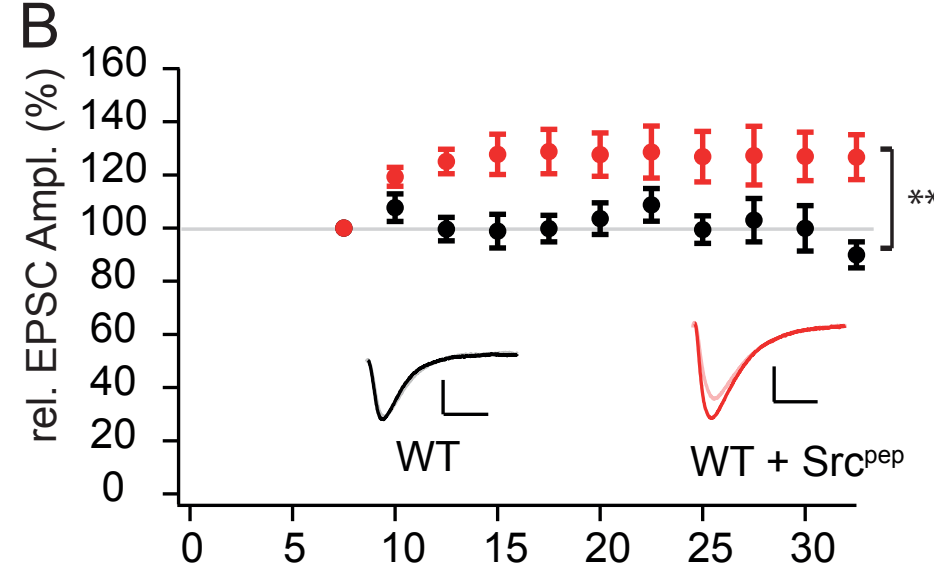

C
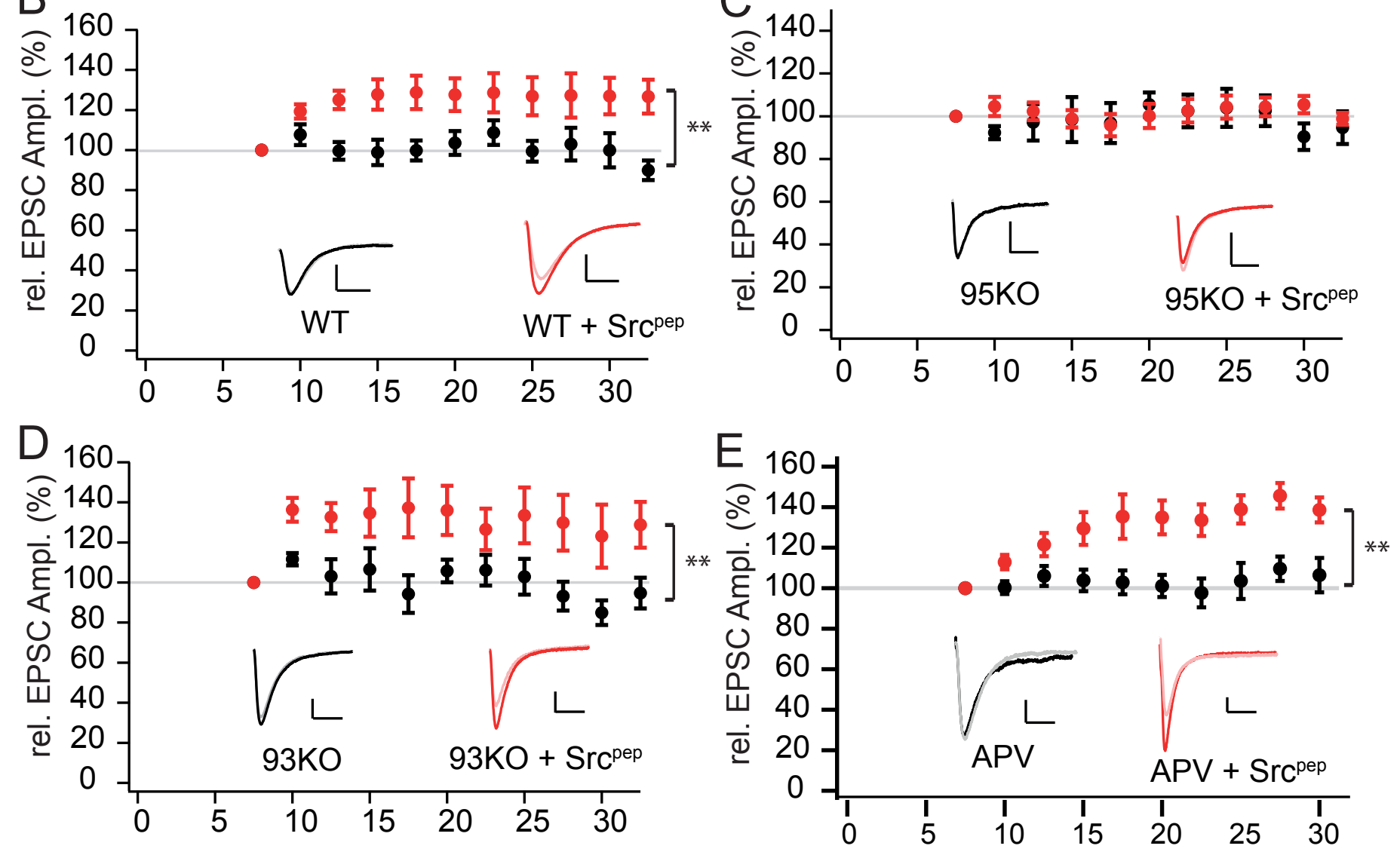

Fig. 4 
A

C

PSD-95

$\mathrm{D}$

shSrc

SrC

bioRxiv preprint doi: https://doi.org/10.1101/2020.10.02.323568; this versignposted October 2, 2020. The copyright holder for this preprint
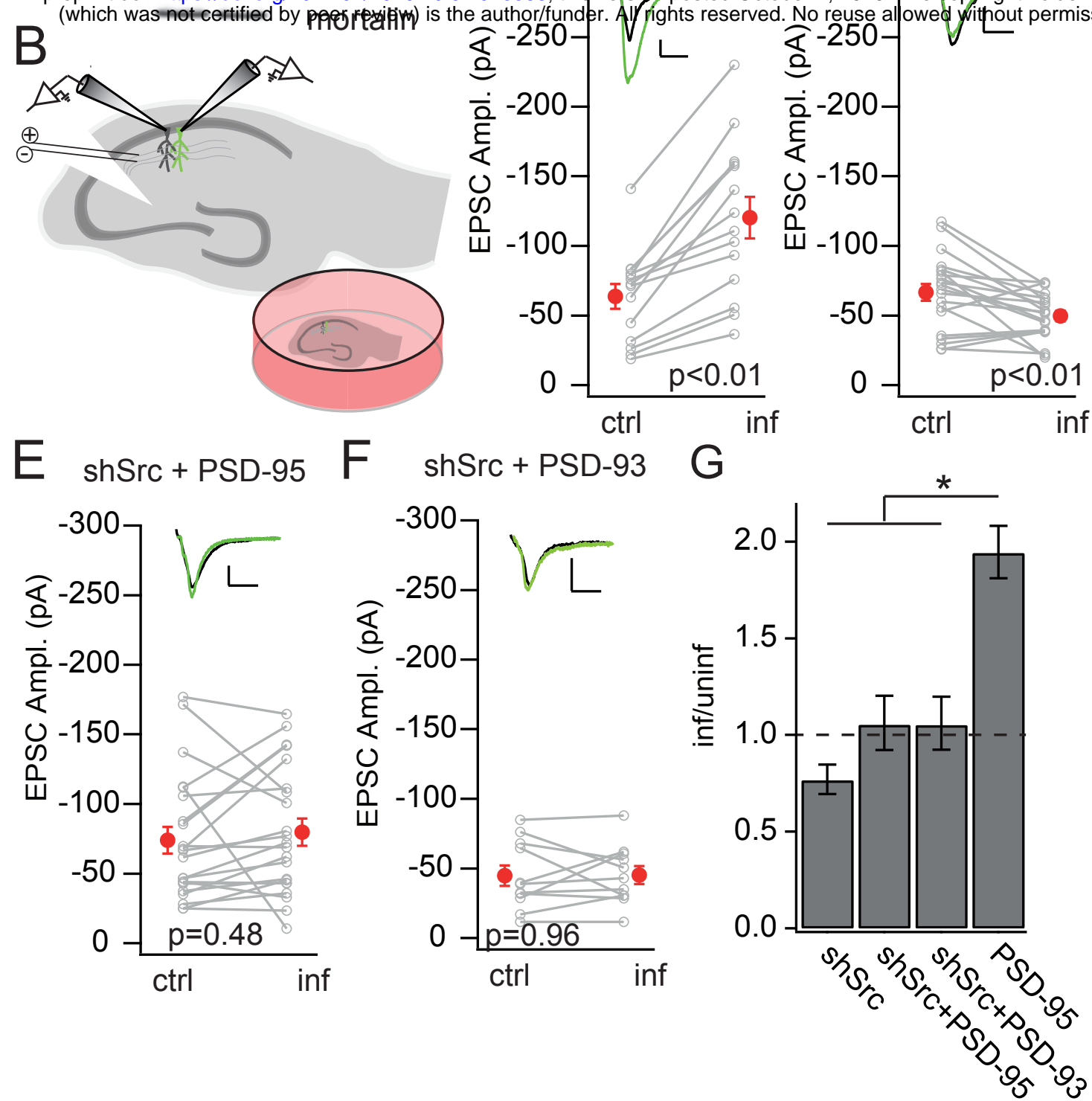

$\mathrm{H}$

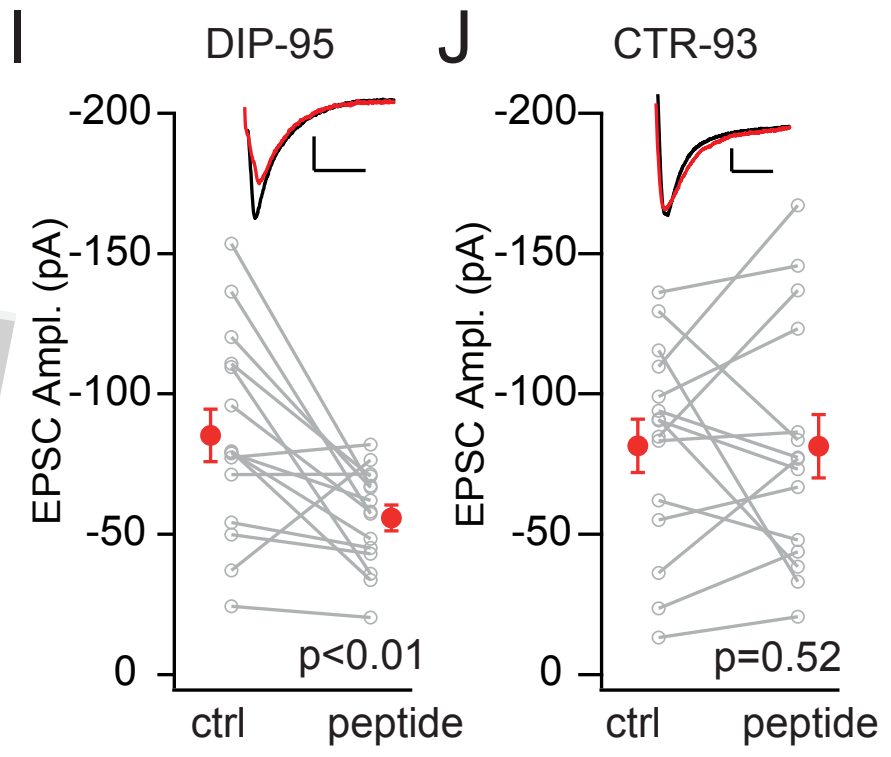

Fig. 5 